\title{
Palladium-Catalyzed Decarboxylative Aza-Michael Addition-Allylation Reactions Between Allyl Carbamates and Activated Olefins. Generation of Quaternary Carbon Adjacent to Secondary Amine Carbon Center
}

Nitin T. Patil, Zhibao Huo and Yoshinori Yamamoto*

Department of Chemistry, Graduate School of Science, Tohoku University, Sendai 980-8578, Japan

\section{Contents of Supporting Information}

(27 Pages)

Page S-1: Title of the paper, author's name and address along with the contents.

Page S-2: Characterization data of compounds

Page S-7: ${ }^{1} \mathrm{H}$ NMR spectra of compounds $\mathbf{3 e}$ and $\mathbf{3 g}$

Page S-8: ${ }^{1} \mathrm{H}$ NMR spectra of compounds $\mathbf{3 h}$ and $\mathbf{3 i}$

Page S-9: ${ }^{1} \mathrm{H}$ NMR spectra of compounds $\mathbf{3 j}$ and $\mathbf{3 k}$

Page S-10: ${ }^{1} \mathrm{H}$ NMR spectra of compounds $\mathbf{3 l}$ and $\mathbf{3 m}$

Page S-11: ${ }^{1} \mathrm{H}$ NMR spectra of compounds $\mathbf{5 a}$ and $\mathbf{5 b}$

Page S-12: ${ }^{1} \mathrm{H}$ NMR spectra of compounds $\mathbf{5 c}$ and $\mathbf{5 d}$

Page S-13: ${ }^{1} \mathrm{H}$ NMR spectra of compounds $\mathbf{5 e}$ and $\mathbf{5 f}$

Page S-14: ${ }^{1}$ H NMR spectra of compounds $\mathbf{5 g}$ and $\mathbf{5 h}$

Page S-15: ${ }^{1} \mathrm{H}$ NMR spectra of compounds $\mathbf{5 j}$ and $\mathbf{5 k}$

Page S-16: ${ }^{1} \mathrm{H}$ NMR spectra of compounds $\mathbf{5 l}$ and $\mathbf{5 m}$

Page S-17: ${ }^{1} \mathrm{H}$ NMR spectra of compounds $\mathbf{5 n}$

Page S-18: X-Ray structure of 5n

(3e). ${ }^{1} \mathrm{H}$ NMR $\left(400 \mathrm{MHz}, \mathrm{CDCl}_{3}\right) \delta 1.08$ (br s, 3H), $2.58(\mathrm{ddd}, J=14.0,7.0,1.0 \mathrm{~Hz}, 1 \mathrm{H}), 2.65$ (ddd, 
$J=14.0,7.0,1.0 \mathrm{~Hz}, 1 \mathrm{H}), 4.13(\mathrm{q}, J=7.0 \mathrm{~Hz}, 2 \mathrm{H}), 5.26(\mathrm{dt}, J=17.0,1.0 \mathrm{~Hz}, 1 \mathrm{H}), 5.37(\mathrm{dt}, J=10.0$, $1.0 \mathrm{~Hz}, 1 \mathrm{H}), 5.95-5.83(\mathrm{~m}, 2 \mathrm{H}), 6.89-6.84(\mathrm{~m}, 2 \mathrm{H}), 7.35-7.20(\mathrm{~m}, 8 \mathrm{H}) .{ }^{13} \mathrm{C} \mathrm{NMR}\left(100 \mathrm{MHz}, \mathrm{CDCl}_{3}\right)$ $\delta 14.4,41.2,42.2$, 62.9, 63.8, 114.3, 114.4, 123.6, 128.1, 128.7, 128.7, 128.8, 129.6, 129.7, 130.5, 133.1, 137.1, 156.4. IR (neat) 2981, 2248, 1699, 1596, 1456, 1292, 937, $700 \mathrm{~cm}^{-1}$. ESI Calcd for $\mathrm{C}_{22} \mathrm{H}_{21} \mathrm{~N}_{3} \mathrm{O}_{2}(\mathrm{M}+\mathrm{Na})$ 382.1526. Found 382.1525.

(3g). ${ }^{1} \mathrm{H}$ NMR (400MHz, $\left.\mathrm{CDCl}_{3}\right) \delta$ 2.00-0.50 (m, 13H), $2.65(\mathrm{ddd}, J=14.0,7.0,1.0 \mathrm{~Hz}, 1 \mathrm{H}), 2.78$ (ddd, $J=14.0,7.0,1.0 \mathrm{~Hz}, 1 \mathrm{H}), 3.35(\mathrm{br} \mathrm{s}, 1 \mathrm{H}), 4.22(\mathrm{q}, J=7.0 \mathrm{~Hz}, 2 \mathrm{H}), 5.31$ (dt, $J=17.0,1.0 \mathrm{~Hz}$, $1 \mathrm{H}), 5.36(\mathrm{~s}, 1 \mathrm{H}), 5.39$ (dt, $J=10.0,1.0 \mathrm{~Hz}, 1 \mathrm{H}), 5.92(\mathrm{ddt}, J=17.0,10.0,1.0 \mathrm{~Hz}, 1 \mathrm{H}), 7.57-7.32(\mathrm{~m}$, 5H). ${ }^{13} \mathrm{C} \mathrm{NMR}\left(100 \mathrm{MHz}, \mathrm{CDCl}_{3}\right) \delta 14.5,25.3,25.8,26.2,38.5,38.9,40.9,62.1,71.8,123.5,126.7$, 128.7, 129.1, 129.4, 130.1, 137.2, 142.2, 167.1. IR (neat) 2933, 2250, 1693, 1606, 1440, 1288, 908, $702 \mathrm{~cm}^{-1}$. ESI Calcd for $\mathrm{C}_{22} \mathrm{H}_{27} \mathrm{~N}_{3} \mathrm{O}_{2}(\mathrm{M}+\mathrm{Na})$ 388.1995. Found 388.1996.

(3h). ${ }^{1} \mathrm{H}$ NMR $\left(400 \mathrm{MHz}, \mathrm{CDCl}_{3}\right) \delta 1.07(\mathrm{t}, J=7.0 \mathrm{~Hz}, 3 \mathrm{H}), 2.56(\mathrm{ddd}, J=14.0,7.0,1.0 \mathrm{~Hz}, 1 \mathrm{H})$, $2.63(\mathrm{ddd}, J=14.0,7.0,1.0 \mathrm{~Hz}, 1 \mathrm{H}), 3.75(\mathrm{~s}, 3 \mathrm{H}), 4.09$ (q, $J=7.0 \mathrm{~Hz}, 2 \mathrm{H}), 5.25$ (dt, $J=17.0,1.0 \mathrm{~Hz}$, $1 \mathrm{H}), 5.36(\mathrm{dt}, J=10.0,1.0 \mathrm{~Hz}, 1 \mathrm{H}), 5.83(\mathrm{~s}, 1 \mathrm{H}), 5.86$ (ddt, $J=17.0,10.0,1.0 \mathrm{~Hz}, 1 \mathrm{H}), 6.77(\mathrm{~d}, J=$ $8.0 \mathrm{~Hz}, 2 \mathrm{H}), 6.88(\mathrm{~d}, J=8.0 \mathrm{~Hz}, 1 \mathrm{H}), 6.95(\mathrm{~d}, J=8.0 \mathrm{~Hz}, 1 \mathrm{H}), 7.32-7.17(\mathrm{~m}, 4 \mathrm{H}), 7.83(\mathrm{~d}, J=8.0 \mathrm{~Hz}$, 1H). ${ }^{13} \mathrm{C}$ NMR $\left(100 \mathrm{MHz}, \mathrm{CDCl}_{3}\right) \delta 14.3,41.1,42.4,55.2,62.8,63.3,114.1,114.4,114.5,115.1$, 123.5, 124.9, 128.3, 128.7, 128.7, 130.7, 131.1, 133.4, 160.4. IR (neat) 2976, 2225, 1705, 1610, $1463,1294,1022,755 \mathrm{~cm}^{-1}$. ESI Calcd for $\mathrm{C}_{23} \mathrm{H}_{23} \mathrm{~N}_{3} \mathrm{O}_{3}(\mathrm{M}+\mathrm{Na}) 412.1632$. Found 412.1631.

(3i). ${ }^{1} \mathrm{H}$ NMR (400MHz, $\left.\mathrm{CDCl}_{3}\right) \delta 1.08(\mathrm{t}, J=7.0 \mathrm{~Hz}, 3 \mathrm{H}), 2.62(\mathrm{ddd}, J=14.0,7.0,1.0 \mathrm{~Hz}, 1 \mathrm{H})$, 2.71 (ddd, $J=14.0,7.0,1.0 \mathrm{~Hz}, 1 \mathrm{H}), 4.13(\mathrm{q}, J=7.0 \mathrm{~Hz}, 2 \mathrm{H}), 5.28(\mathrm{dt}, J=17.0,1.0 \mathrm{~Hz}, 1 \mathrm{H}), 5.37$ (dt, $J=10.0,1.0 \mathrm{~Hz}, 1 \mathrm{H}), 5.86(\mathrm{ddt}, J=17.0,10.0,1.0 \mathrm{~Hz}, 1 \mathrm{H}), 6.14(\mathrm{br} \mathrm{s}, 1 \mathrm{H}), 6.32$ (m, 1H), $6.41(\mathrm{~d}, J$ $=3.0 \mathrm{~Hz}, 1 \mathrm{H}), 6.89-6.81(\mathrm{~m}, 2 \mathrm{H}), 7.38-7.19(\mathrm{~m}, 4 \mathrm{H}) .{ }^{13} \mathrm{C} \mathrm{NMR}\left(100 \mathrm{MHz}, \mathrm{CDCl}_{3}\right) \delta 14.3,40.6$, 41.4, 57.6, 63.0, 110.9, 111.9, 113.6, 113.7, 123.8, 127.9, 128.8, 128.8, 129.9, 129.9, 143.2, 146.1, 155.4. IR (neat) 2977, 2254, 1708, 1596, 1444, 1298, 914, $732 \mathrm{~cm}^{-1}$. ESI Calcd for $\mathrm{C}_{20} \mathrm{H}_{19} \mathrm{~N}_{3} \mathrm{O}_{3}$ (M+Na) 372.1319. Found 372.1319.

(3j). ${ }^{1} \mathrm{H}$ NMR $\left(400 \mathrm{MHz}, \mathrm{CDCl}_{3}\right) \delta 1.18(\mathrm{t}, J=7.0 \mathrm{~Hz}, 3 \mathrm{H}), 1.36(\mathrm{t}, J=7.0 \mathrm{~Hz}, 3 \mathrm{H}), 2.74(\mathrm{ddd}, J=$ 14.0, 7.0, 1.0 Hz, 1H), 2.79 (ddd, $J=14.0,7.0,1.0 \mathrm{~Hz}, 1 \mathrm{H}), 3.76(\mathrm{dq}, J=10.0,7.0 \mathrm{~Hz}, 1 \mathrm{H}), 4.01$ (dq, $J=10.0,7.0 \mathrm{~Hz}, 1 \mathrm{H}), 4.22(\mathrm{dq}, J=10.0,7.0 \mathrm{~Hz}, 2 \mathrm{H}), 5.37(\mathrm{dt}, J=17.0,1.0 \mathrm{~Hz}, 1 \mathrm{H}), 5.41(\mathrm{dt}, J=$ 10.0, $1.0 \mathrm{~Hz}, 1 \mathrm{H}), 5.87$ (ddt, $J=17.0,10.0,1.0 \mathrm{~Hz}, 1 \mathrm{H}), 6.01$ (br s, $1 \mathrm{H}), 7.44-7.29(\mathrm{~m}, 5 \mathrm{H}) .{ }^{13} \mathrm{C}$ $\operatorname{NMR}\left(100 \mathrm{MHz}, \mathrm{CDCl}_{3}\right) \delta 14.2,14.5,40.0,42.3,62.9,65.3,84.6,111.7,112.9,123.3,128.3,129.0$, 129.1, 130.1, 134.7, 150.6. IR (neat) 2981, 2254, 1710, 1596, 1464, 1301, 914, $732 \mathrm{~cm}^{-1}$. ESI Calcd for $\mathrm{C}_{18} \mathrm{H}_{21} \mathrm{~N}_{3} \mathrm{O}_{3}(\mathrm{M}+\mathrm{Na}) 350.1475$. Found 350.1474.

(3k). ${ }^{1} \mathrm{H}$ NMR $\left(400 \mathrm{MHz}, \mathrm{CDCl}_{3}\right) \delta 0.90(\mathrm{t}, J=7.0 \mathrm{~Hz}, 3 \mathrm{H}), 1.61-1.05(\mathrm{~m}, 9 \mathrm{H}), 2.70(\mathrm{ddd}, J=14.0$, 
7.0, 1.0 Hz, 1H), 2.77 (ddd, $J=14.0,7.0,1.0 \mathrm{~Hz}, 1 \mathrm{H}), 4.17$ (q, $J=7.0 \mathrm{~Hz}, 2 \mathrm{H}), 4.90$ (br s, 1H), 5.37 $(\mathrm{dt}, J=17.0,1.0 \mathrm{~Hz}, 1 \mathrm{H}), 5.41(\mathrm{dt}, J=10.0,1.0 \mathrm{~Hz}, 1 \mathrm{H}), 5.91(\mathrm{ddt}, J=17.0,10.0,1.0 \mathrm{~Hz}, 1 \mathrm{H})$, 7.42-7.20 (m, 5H). ${ }^{13} \mathrm{C}$ NMR $\left(100 \mathrm{MHz}, \mathrm{CDCl}_{3}\right) \delta 13.8,14.3,15.2,22.2,28.4,40.6,43.0,62.7,65.8$, 113.7, 114.1, 123.3, 128.5, 128.7, 128.9, 129.2, 129.6, 156.6. IR (neat) 2962, 2246, 1705, 1596, $1465,1299,937,702 \mathrm{~cm}^{-1}$. ESI Calcd for $\mathrm{C}_{20} \mathrm{H}_{25} \mathrm{~N}_{3} \mathrm{O}_{2}(\mathrm{M}+\mathrm{Na}) 362.1839$. Found 362.1837.

(31). ${ }^{1} \mathrm{H}$ NMR (400MHz, $\mathrm{CDCl}_{3}$ ) $\delta 1.11$ (br s, 6H), $1.16(\mathrm{t}, J=7.0 \mathrm{~Hz}, 3 \mathrm{H}), 2.84-2.59(\mathrm{~m}, 2 \mathrm{H}), 3.02$ (br s, 1H), 3.35 (br s, 1H), 4.20 (br s, 2H), 5.38-5.27 (m, 2H), 5.89 (br s, 1H), 7.36-7.18 (m, 5H). ${ }^{13} \mathrm{C}$ NMR (100 MHz, $\left.\mathrm{CDCl}_{3}\right) \delta 14.4,21.6,31.1,42.5,43.3,62.6,72.6,114.5,115.1,123.3,126.5,126.8$, 128.9, 129.0, 143.8, 154.9. IR (neat) 2977, 2246, 1705, 1596, 1465, 1208, 935, $698 \mathrm{~cm}^{-1}$. ESI Calcd for $\mathrm{C}_{19} \mathrm{H}_{23} \mathrm{~N}_{3} \mathrm{O}_{2}(\mathrm{M}+\mathrm{Na})$ 348.1682. Found 348.1682.

(3m). ${ }^{1} \mathrm{H}$ NMR (400MHz, $\left.\mathrm{CDCl}_{3}\right) \delta 1.25(\mathrm{~s}, 9 \mathrm{H}), 1.29(\mathrm{t}, J=7.0 \mathrm{~Hz}, 3 \mathrm{H}), 2.92(\mathrm{ddd}, J=14.0,7.0$, $1.0 \mathrm{~Hz}, 1 \mathrm{H}), 3.07$ (ddd, $J=14.0,7.0,1.0 \mathrm{~Hz}, 1 \mathrm{H}), 3.75(\mathrm{~s}, 1 \mathrm{H}), 4.33-4.19$ (m, 2H), 5.45 (dt, $J=17.0$, $1.0 \mathrm{~Hz}, 1 \mathrm{H}), 5.52(\mathrm{dt}, J=10.0,1.0 \mathrm{~Hz}, 1 \mathrm{H}), 6.10$ (ddt, $J=17.0,10.0,1.0 \mathrm{~Hz}, 1 \mathrm{H}), 7.42-7.22(\mathrm{~m}, 5 \mathrm{H})$. ${ }^{13} \mathrm{C} \mathrm{NMR}\left(100 \mathrm{MHz}, \mathrm{CDCl}_{3}\right) \delta 14.4,29.4,40.7,41.3,43.8,62.5,74.5,115.5,116.2,123.8,126.6$, 126.7, 128.8, 129.0, 144.9, 155.3. IR (KBr) 2981, 2246, 1693, 1596, 1471, 1270, 904, $696 \mathrm{~cm}^{-1}$. ESI Calcd for $\mathrm{C}_{20} \mathrm{H}_{25} \mathrm{~N}_{3} \mathrm{O}_{2}(\mathrm{M}+\mathrm{Na}) 362.1839$. Found 362.1840 .

(5a). ${ }^{1} \mathrm{H}$ NMR (400MHz, $\left.\mathrm{CDCl}_{3}\right) \delta 1.14(\mathrm{t}, J=7.0 \mathrm{~Hz}, 3 \mathrm{H}), 2.22(\mathrm{~s}, 3 \mathrm{H}), 2.70$ (ddd, $J=14.0,7.0,1.0$ $\mathrm{Hz}, 1 \mathrm{H}), 2.83(\mathrm{ddd}, J=14.0,7.0,1.0 \mathrm{~Hz}, 1 \mathrm{H}), 4.14(\mathrm{q}, J=7.0 \mathrm{~Hz}, 2 \mathrm{H}), 5.28$ (dt, $J=17.0,1.0 \mathrm{~Hz}$, $1 \mathrm{H}), 5.32(\mathrm{dt}, J=10.0,1.0 \mathrm{~Hz}, 1 \mathrm{H}), 5.88$ (ddt, $J=17.0,10.0,1.0 \mathrm{~Hz}, 1 \mathrm{H}), 6.56$ (br s, 1H), 6.90 (d, $J$ $=8.0 \mathrm{~Hz}, 1 \mathrm{H}), 7.40-7.15(\mathrm{~m}, 7 \mathrm{H}) .{ }^{13} \mathrm{C} \mathrm{NMR}\left(100 \mathrm{MHz}, \mathrm{CDCl}_{3}\right) \delta 14.2,20.2,38.3,52.4,62.9,85.9$, 115.3, 117.3, 117.6, 122.3, 126.8, 127.9, 128.8, 128.8, 129.3, 130.3, 131.9, 137.9, 155.4, 156.3, 182.9. IR (neat) $3390,2981,2248,1699,1640,1488,1199,935,732 \mathrm{~cm}^{-1}$. ESI Calcd for $\mathrm{C}_{23} \mathrm{H}_{22} \mathrm{~N}_{2} \mathrm{O}_{4}(\mathrm{M}+\mathrm{Na})$ 413.1472. Found 413.1473.

(5b). ${ }^{1} \mathrm{H}$ NMR (400MHz, $\left.\mathrm{CDCl}_{3}\right) \delta 1.09$ (t, $\left.J=7.0 \mathrm{~Hz}, 3 \mathrm{H}\right), 2.65$ (ddd, $J=14.0,7.0,1.0 \mathrm{~Hz}, 1 \mathrm{H}$ ), $2.75(\mathrm{ddd}, J=14.0,7.0,1.0 \mathrm{~Hz}, 1 \mathrm{H}), 4.09(\mathrm{q}, J=7.0 \mathrm{~Hz}, 2 \mathrm{H}), 5.24(\mathrm{dt}, J=17.0,1.0 \mathrm{~Hz}, 1 \mathrm{H}), 5.30$ (dt, $J=10.0,1.0 \mathrm{~Hz}, 1 \mathrm{H}), 5.82(\mathrm{ddt}, J=17.0,10.0,1.0 \mathrm{~Hz}, 1 \mathrm{H}), 6.51(\mathrm{br} \mathrm{s}, 1 \mathrm{H}), 6.87(\mathrm{~d}, J=9.0 \mathrm{~Hz}, 1 \mathrm{H})$, 7.25-7.13 (m, 5H), 7.52 (dd, $J=9.0,2.0 \mathrm{~Hz}, 1 \mathrm{H}), 7.66$ (br s, $1 \mathrm{H}) .{ }^{13} \mathrm{C} \mathrm{NMR}\left(100 \mathrm{MHz}, \mathrm{CDCl}_{3}\right) \delta$ $14.2,38.5,52.5,63.2,86.4,114.9,119.2,119.5,122.8,128.9,128.9,129.0,129.0,129.5,130.1$, 136.4, 139.5, 155.3, 157.1, 181.6. IR (KBr) 3360, 2983, 2254, 1701, 1598, 1465, 1286, 1020, 929 $\mathrm{cm}^{-1}$. ESI Calcd for $\mathrm{C}_{22} \mathrm{H}_{19} \mathrm{BrN}_{2} \mathrm{O}_{4}(\mathrm{M}+\mathrm{Na})$ 477.0420. Found 477.0421.

(5c). ${ }^{1} \mathrm{H}$ NMR $\left(400 \mathrm{MHz}, \mathrm{CDCl}_{3}\right) \delta 1.16(\mathrm{t}, J=7.0 \mathrm{~Hz}, 3 \mathrm{H}), 2.72(\mathrm{ddd}, J=14.0,7.0,1.0 \mathrm{~Hz}, 1 \mathrm{H})$, $2.80(\mathrm{ddd}, J=14.0,7.0,1.0 \mathrm{~Hz}, 1 \mathrm{H}), 4.16(\mathrm{q}, J=7.0 \mathrm{~Hz}, 2 \mathrm{H}), 5.32(\mathrm{dt}, J=17.0,1.0 \mathrm{~Hz}, 1 \mathrm{H}), 5.38(\mathrm{dt}$, $J=10.0,1.0 \mathrm{~Hz}, 1 \mathrm{H}), 5.89$ (ddt, $J=17.0,10.0,1.0 \mathrm{~Hz}, 1 \mathrm{H}), 6.60(\mathrm{br} \mathrm{s}, 1 \mathrm{H}), 7.06(\mathrm{t}, J=7.0 \mathrm{~Hz}, 1 \mathrm{H})$, 
7.40-7.25 (m, 4H), 7.57-7.52 (m, 2H). ${ }^{13} \mathrm{C} \mathrm{NMR}\left(100 \mathrm{MHz}, \mathrm{CDCl}_{3}\right) \delta$ 14.1, 38.5, 52.6, 63.3, 87.2, 114.6, 118.6, 119.6, 123.1, 123.5, 125.1, 127.5, 128.6, 128.9, 129.1, 129.2, 136.2, 152.3, 155.1, 181.1. IR (neat) 3348, 2983, 2252, 1716, 1645, 1456, 1298, $1060 \mathrm{~cm}^{-1}$. ESI Calcd for $\mathrm{C}_{22} \mathrm{H}_{18} \mathrm{Cl}_{2} \mathrm{~N}_{2} \mathrm{O}_{4}(\mathrm{M}+\mathrm{Na})$ 467.0536. Found 467.0538.

(5d). ${ }^{1} \mathrm{H}$ NMR $\left(400 \mathrm{MHz}, \mathrm{CDCl}_{3}\right) \delta 1.16(\mathrm{t}, J=7.0 \mathrm{~Hz}, 3 \mathrm{H}), 2.73(\mathrm{ddd}, J=14.0,7.0,1.0 \mathrm{~Hz}, 1 \mathrm{H})$, $2.86(\mathrm{ddd}, J=14.0,7.0,1.0 \mathrm{~Hz}, 1 \mathrm{H}), 4.17(\mathrm{q}, J=7.0 \mathrm{~Hz}, 2 \mathrm{H}), 5.30(\mathrm{dt}, J=17.0,1.0 \mathrm{~Hz}, 1 \mathrm{H}), 5.36(\mathrm{dt}$, $J=10.0,1.0 \mathrm{~Hz}, 1 \mathrm{H}), 5.91(\mathrm{ddt}, J=17.0,10.0,1.0 \mathrm{~Hz}, 1 \mathrm{H}), 6.65(\mathrm{br} \mathrm{s}, 1 \mathrm{H}), 6.95(\mathrm{t}, J=8.0 \mathrm{~Hz}, 1 \mathrm{H})$, $7.02(\mathrm{~d}, J=8.0 \mathrm{~Hz}, 1 \mathrm{H}), 7.30-7.15(\mathrm{~m}, 5 \mathrm{H}), 7.50(\mathrm{t}, J=8.0 \mathrm{~Hz}, 1 \mathrm{H}), 7.60(\mathrm{~d}, J=8.0 \mathrm{~Hz}, 1 \mathrm{H}) .{ }^{13} \mathrm{C}$ NMR $\left(100 \mathrm{MHz}, \mathrm{CDCl}_{3}\right) \delta 14.2,38.4,52.6,63.0,85.9,115.2,117.4,117.9,122.3,122.4,127.3$, 128.8, 128.9, 129.2, 130.3, 136.9, 136.9, 155.3, 158.2, 182.7. IR (KBr) 3332, 2981, 2248, 1699, 1606, 1461, 1292, 985, $702 \mathrm{~cm}^{-1}$. ESI Calcd for $\mathrm{C}_{22} \mathrm{H}_{20} \mathrm{~N}_{2} \mathrm{O}_{4}(\mathrm{M}+\mathrm{Na}) 399.1315$. Found 399.1316.

(5e). ${ }^{1} \mathrm{H}$ NMR (400MHz, $\left.\mathrm{CDCl}_{3}\right) \delta 1.07$ (t, $\left.J=7.0 \mathrm{~Hz}, 3 \mathrm{H}\right), 2.57$ (ddd, $\left.J=14.0,7.0,1.0 \mathrm{~Hz}, 1 \mathrm{H}\right)$, $2.96(\mathrm{ddd}, J=14.0,7.0,1.0 \mathrm{~Hz}, 1 \mathrm{H}), 4.04(\mathrm{q}, J=7.0 \mathrm{~Hz}, 2 \mathrm{H}), 5.16(\mathrm{dt}, J=10.0,1.0 \mathrm{~Hz}, 1 \mathrm{H}), 5.17$ (dt, $J=17.0,1.0 \mathrm{~Hz}, 1 \mathrm{H}), 5.71(\mathrm{ddt}, J=17.0,10.0,1.0 \mathrm{~Hz}, 1 \mathrm{H}), 6.24(\mathrm{br} \mathrm{s}, 1 \mathrm{H}), 7.10(\mathrm{t}, J=8.0 \mathrm{~Hz}, 2 \mathrm{H})$, 7.37-7.28 (m, 5H), $7.56(\mathrm{t}, J=8.0 \mathrm{~Hz}, 1 \mathrm{H}), 7.91(\mathrm{~d}, J=8.0 \mathrm{~Hz}, 1 \mathrm{H}), 9.65(\mathrm{br} \mathrm{s}, 1 \mathrm{H}) .{ }^{13} \mathrm{C} \mathrm{NMR}(100$ $\left.\mathrm{MHz}, \mathrm{CDCl}_{3}\right) \delta 14.2,35.6,62.7,62.7,89.8,118.3,120.7,120.9,122.5,127.5,128.7,129.1,129.4$, $130.5,136.3,139.3,155.4,158.5,187.6,194.9$. IR (neat) 3363, 2981, 1716, 1606, 1463, 1218, 810 $\mathrm{cm}^{-1}$. ESI Calcd for $\mathrm{C}_{22} \mathrm{H}_{21} \mathrm{NO}_{5}(\mathrm{M}+\mathrm{Na})$ 402.1312. Found 402.1313.

(5f). ${ }^{1} \mathrm{H} \mathrm{NMR}\left(400 \mathrm{MHz}, \mathrm{CDCl}_{3}\right) \delta 1.03(\mathrm{t}, J=7.0 \mathrm{~Hz}, 3 \mathrm{H}), 2.66(\mathrm{ddd}, J=14.0,7.0,1.0 \mathrm{~Hz}, 1 \mathrm{H})$, 2.84 (ddd, $J=14.0,7.0,1.0 \mathrm{~Hz}, 1 \mathrm{H}), 3.99$ (q, $J=7.0 \mathrm{~Hz}, 2 \mathrm{H}), 5.17$ (dt, $J=17.0,1.0 \mathrm{~Hz}, 1 \mathrm{H}), 5.18$ (dt, $J=10.0,1.0 \mathrm{~Hz}, 1 \mathrm{H}), 5.67$ (ddt, $J=17.0,10.0,1.0 \mathrm{~Hz}, 1 \mathrm{H}), 6.09$ (br s, 1H), 7.43-7.34 (m, 5H), 7.90 $(\mathrm{dd}, J=7.0,3.0 \mathrm{~Hz}, 1 \mathrm{H}), 7.95(\mathrm{dd}, J=7.0,3.0 \mathrm{~Hz}, 1 \mathrm{H}), 9.72$ (br s, $1 \mathrm{H}) .{ }^{13} \mathrm{C} \mathrm{NMR}\left(100 \mathrm{MHz}, \mathrm{CDCl}_{3}\right)$ $\delta 14.5,21.0,29.3,63.1,96.7,117.1,118.2,118.6,121.9,123.7,128.6,128.9,129.1,129.2,129.5$, 133.3, 152.8, 156.0, 185.1, 185.8. IR (KBr) 3404, 3082, 2868, 1710, 1625, 1465, 1244, 867, 696 $\mathrm{cm}^{-1}$. ESI Calcd for $\mathrm{C}_{22} \mathrm{H}_{19} \mathrm{FN}_{2} \mathrm{O}_{7}(\mathrm{M}+\mathrm{Na})$ 465.1069. Found 465.1067.

(5g). ${ }^{1} \mathrm{H}$ NMR (400MHz, $\left.\mathrm{CDCl}_{3}\right) \delta 1.12(\mathrm{t}, J=7.0 \mathrm{~Hz}, 3 \mathrm{H}), 2.42(\mathrm{ddd}, J=14.0,7.0,1.0 \mathrm{~Hz}, 1 \mathrm{H})$, $2.68(\mathrm{ddd}, J=14.0,7.0,1.0 \mathrm{~Hz}, 1 \mathrm{H}), 4.20-4.07(\mathrm{~m}, 2 \mathrm{H}), 5.13(\mathrm{dt}, J=17.0,1.0 \mathrm{~Hz}, 1 \mathrm{H}), 5.37(\mathrm{dt}, J=$ 10.0, $1.0 \mathrm{~Hz}, 1 \mathrm{H}), 5.94$ (ddt, $J=17.0,10.0,1.0 \mathrm{~Hz}, 1 \mathrm{H}), 6.97$ (br s, 1H), 7.45-7.24 (m, 9H). ${ }^{13} \mathrm{C}$ NMR (100 MHz, $\left.\mathrm{CDCl}_{3}\right) \delta 14.2,38.9,50.4,50.4,62.7,115.9,116.6,116.9,122.9,125.7,125.9$, $128.5,129.1,129.1,129.5,131.2,150.6,156.3,161.3,190.1$. IR (KBr) 3527, 2991, 2252, 1705 , $1639,1452,1112,904,765 \mathrm{~cm}^{-1}$. ESI Calcd for $\mathrm{C}_{22} \mathrm{H}_{20} \mathrm{~N}_{2} \mathrm{O}_{4}(\mathrm{M}+\mathrm{Na}) 399.1315$. Found 399.1317.

(5h). ${ }^{1} \mathrm{H}$ NMR $\left(400 \mathrm{MHz}, \mathrm{CDCl}_{3}\right) \delta 1.20(\mathrm{t}, J=7.0 \mathrm{~Hz}, 3 \mathrm{H}), 2.70-2.44(\mathrm{~m}, 6 \mathrm{H}), 4.11$ (q, $J=7.0 \mathrm{~Hz}$, $2 \mathrm{H}), 5.11(\mathrm{dt}, J=17.0,1.0 \mathrm{~Hz}, 1 \mathrm{H}), 5.14(\mathrm{dt}, J=10.0,1.0 \mathrm{~Hz}, 1 \mathrm{H}), 5.74-5.59(\mathrm{~m}, 2 \mathrm{H}), 6.02-5.96(\mathrm{~m}$, 
1H), 6.45 (br s, 1H), $6.94(\mathrm{t}, J=7.0 \mathrm{~Hz}, 1 \mathrm{H}), 7.59-7.16(\mathrm{~m}, 4 \mathrm{H}) .{ }^{13} \mathrm{C} \mathrm{NMR}\left(100 \mathrm{MHz}, \mathrm{CDCl}_{3}\right) \delta 14.5$, 26.1, 35.8, 41.4, 49.3, 61.1, 61.2, 63.1, 118.7, 123.2, 128.5, 128.9, 130.7, 131.9, 137.9, 153.6, 182.1. IR (neat) 3328, 2981, 2236, 1722, 1598, 1444, 1224, $698 \mathrm{~cm}^{-1}$. ESI Calcd for $\mathrm{C}_{19} \mathrm{H}_{22} \mathrm{~N}_{2} \mathrm{O}_{3}(\mathrm{M}+\mathrm{Na})$ 349.1523. Found 349.1525.

(5j). ${ }^{1} \mathrm{H}$ NMR $\left(400 \mathrm{MHz}, \mathrm{CDCl}_{3}\right) \delta$ 1.04-1.45 (m, 13H), 2.67 (br s, 1H), 2.84-2.75 (m, 1H), 3.47 (br $\mathrm{s}, 1 \mathrm{H}), 4.10(\mathrm{q}, J=7.0 \mathrm{~Hz}, 2 \mathrm{H}), 4.50$ (br s, 1H), 5.44-5.28 (m, 2H), 6.05-5.91 (m, 1H), 6.94 (br s, $1 \mathrm{H}), 7.13-7.04(\mathrm{~m}, 1 \mathrm{H}), 7.52$ (br s, $1 \mathrm{H}), 7.98-7.90(\mathrm{~m}, 1 \mathrm{H}) .{ }^{13} \mathrm{C} \mathrm{NMR}\left(100 \mathrm{MHz}, \mathrm{CDCl}_{3}\right) \delta 14.2$, 14.6, 24.8, 25.4, 25.9, 33.4, 60.3, 62.2, 85.8, 115.6, 118.1, 118.9, 121.9, 122.1, 123.1, 127.7, 130.3, 132.1, 156.9, 175.6. IR (neat) 3332, 2979, 2256, 1699, 1608, 1468, 1228, $1058 \mathrm{~cm}^{-1}$. ESI Calcd for $\mathrm{C}_{22} \mathrm{H}_{26} \mathrm{~N}_{2} \mathrm{O}_{4}(\mathrm{M}+\mathrm{Na})$ 405.1785. Found 405.1787.

(5k). ${ }^{1} \mathrm{H}$ NMR $\left(400 \mathrm{MHz}, \mathrm{CDCl}_{3}\right) \delta 1.30-1.17(\mathrm{~m}, 3 \mathrm{H}), 2.71-2.54(\mathrm{~m}, 1 \mathrm{H}), 2.91-2.77(\mathrm{~m}, 1 \mathrm{H}), 3.11(\mathrm{~s}$, $3 \mathrm{H}), 4.15(\mathrm{q}, J=7.0 \mathrm{~Hz}, 2 \mathrm{H}), 5.18(\mathrm{dt}, J=17.0,1.0 \mathrm{~Hz}, 1 \mathrm{H}), 5.19(\mathrm{dt}, J=10.0,1.0 \mathrm{~Hz}, 1 \mathrm{H}), 5.82$ (ddt, $J=17.0,10.0,1.0 \mathrm{~Hz}, 1 \mathrm{H}), 6.15$ (br s, $1 \mathrm{H}), 7.01$ (d, $J=8.0 \mathrm{~Hz}, 1 \mathrm{H}), 7.07$ (t, $J=8.0 \mathrm{~Hz}, 1 \mathrm{H})$, $7.53(\mathrm{t}, J=8.0 \mathrm{~Hz}, 1 \mathrm{H}), 7.86(\mathrm{~d}, J=8.0 \mathrm{~Hz}, 1 \mathrm{H}) .{ }^{13} \mathrm{C} \mathrm{NMR}\left(100 \mathrm{MHz}, \mathrm{CDCl}_{3}\right) \delta 14.5,30.0,30.1$, $50.8,62.9,85.3,115.5,117.9,118.1,122.9,128.4,128.4,129.5,137.5,159.4,165.6,183.8$. IR (neat) $3390,2981,2235,1705,1608,1463,1222,931,765 \mathrm{~cm}^{-1}$. ESI Calcd for $\mathrm{C}_{17} \mathrm{H}_{18} \mathrm{~N}_{2} \mathrm{O}_{4}(\mathrm{M}+\mathrm{Na})$ 337.1159. Found 337.1159.

(5l). ${ }^{1} \mathrm{H}$ NMR (400MHz, $\left.\mathrm{CDCl}_{3}\right) \delta 1.09(\mathrm{t}, J=7.0 \mathrm{~Hz}, 3 \mathrm{H}), 2.19(\mathrm{~s}, 3 \mathrm{H}), 2.65(\mathrm{ddd}, J=14.0,7.0,1.0$ $\mathrm{Hz}, 1 \mathrm{H}), 2.78$ (ddd, $J=14.0,7.0,1.0 \mathrm{~Hz}, 1 \mathrm{H}), 4.09$ (q, $J=7.0 \mathrm{~Hz}, 2 \mathrm{H}), 5.23$ (dt, $J=17.0,1.0 \mathrm{~Hz}$, $1 \mathrm{H}), 5.28(\mathrm{dt}, J=10.0,1.0 \mathrm{~Hz}, 1 \mathrm{H}), 5.83$ (ddt, $J=17.0,10.0,1.0 \mathrm{~Hz}, 1 \mathrm{H}), 6.56(\mathrm{br} \mathrm{s}, 1 \mathrm{H}), 6.88(\mathrm{t}, J$ $=8.0 \mathrm{~Hz}, 2 \mathrm{H}), 6.94(\mathrm{~d}, J=8.0 \mathrm{~Hz}, 2 \mathrm{H}), 7.05(\mathrm{br} \mathrm{s}, 2 \mathrm{H}), 7.43(\mathrm{t}, J=8.0 \mathrm{~Hz}, 1 \mathrm{H}), 7.53(\mathrm{~d}, J=8.0 \mathrm{~Hz}$, 1H). ${ }^{13} \mathrm{C}$ NMR $\left(100 \mathrm{MHz}, \mathrm{CDCl}_{3}\right) \delta 14.3,21.0,38.3,52.5,62.9,85.9,115.2,117.5,117.9,122.1$, $122.4,127.3,129.3,129.5,130.0,131.1,136.9,138.9,158.3,161.6,182.8$. IR (KBr) 3386, 2981, 2248, 1710, 1606, 1463, 1290, 1016, 777, $657 \mathrm{~cm}^{-1}$. ESI Calcd for $\mathrm{C}_{23} \mathrm{H}_{22} \mathrm{~N}_{2} \mathrm{O}_{4}(\mathrm{M}+\mathrm{Na}) 413.1472$. Found 413.1471.

(5m). ${ }^{1} \mathrm{H} \mathrm{NMR}\left(400 \mathrm{MHz}, \mathrm{CDCl}_{3}\right) \delta 1.17(\mathrm{t}, J=7.0 \mathrm{~Hz}, 3 \mathrm{H}), 2.72(\mathrm{ddd}, J=14.0,7.0,1.0 \mathrm{~Hz}, 1 \mathrm{H})$, 2.86 (ddd, $J=14.0,7.0,1.0 \mathrm{~Hz}, 1 \mathrm{H}), 3.73$ (br s, $3 \mathrm{H}), 4.16$ (q, $J=7.0 \mathrm{~Hz}, 2 \mathrm{H}), 5.30$ (dt, $J=17.0,1.0$ $\mathrm{Hz}, 1 \mathrm{H}), 5.36$ (dt, $J=10.0,1.0 \mathrm{~Hz}, 1 \mathrm{H}), 5.91$ (ddt, $J=17.0,10.0,1.0 \mathrm{~Hz}, 1 \mathrm{H}), 6.67$ (br s, 3H), 6.95 $(\mathrm{t}, J=8.0 \mathrm{~Hz}, 1 \mathrm{H}), 7.00(\mathrm{~d}, J=8.0 \mathrm{~Hz}, 1 \mathrm{H}), 7.14(\mathrm{br} \mathrm{s}, 2 \mathrm{H}), 7.50(\mathrm{t}, J=8.0 \mathrm{~Hz}, 1 \mathrm{H}), 7.59$ (d, $J=8.0$ $\mathrm{Hz}, 1 \mathrm{H}) .{ }^{13} \mathrm{C} \mathrm{NMR}\left(100 \mathrm{MHz}, \mathrm{CDCl}_{3}\right) \delta 14.2,38.3,52.6,55.3,62.9,85.9,113.9,115.3,115.3,117.4$, $117.9,122.2,122.4,127.3,128.4,129.2,131.6,136.9,158.2,159.6,182.8$. IR (neat) 3388, 2981, 2250, 1716, 1643, 1456, 1288, $753 \mathrm{~cm}^{-1}$. ESI Calcd for $\mathrm{C}_{23} \mathrm{H}_{22} \mathrm{~N}_{2} \mathrm{O}_{5}(\mathrm{M}+\mathrm{Na})$ 429.1421. Found 429.1420 . 
(5n). ${ }^{1} \mathrm{H}$ NMR $\left(400 \mathrm{MHz}, \mathrm{CDCl}_{3}\right) \delta 2.48(\mathrm{ddd}, J=14.0,7.0,1.0 \mathrm{~Hz}, 1 \mathrm{H}), 2.59(\mathrm{ddd}, J=14.0,7.0,1.0$ $\mathrm{Hz}, 1 \mathrm{H}), 5.03$ (dt, $J=17.0,1.0 \mathrm{~Hz}, 1 \mathrm{H}), 5.10(\mathrm{dt}, J=10.0,1.0 \mathrm{~Hz}, 1 \mathrm{H}), 5.35(\mathrm{~d}, J=10.0 \mathrm{~Hz}, 1 \mathrm{H})$, $5.61(\mathrm{dd}, J=17.0,10.0 \mathrm{~Hz}, 1 \mathrm{H}), 5.66(\mathrm{ddt}, J=17.0,10.0,1.0 \mathrm{~Hz}, 1 \mathrm{H}), 6.20$ (d, $J=17.0 \mathrm{~Hz}, 1 \mathrm{H})$, $6.66(\mathrm{t}, J=8.0 \mathrm{~Hz}, 1 \mathrm{H}), 6.71(\mathrm{~d}, J=8.0 \mathrm{~Hz}, 1 \mathrm{H}), 7.02-6.97(\mathrm{~m}, 6 \mathrm{H}), 7.21(\mathrm{t}, J=8.0 \mathrm{~Hz}, 1 \mathrm{H}), 7.31(\mathrm{~d}$, $J=8.0 \mathrm{~Hz}, 1 \mathrm{H}) .{ }^{13} \mathrm{C} \mathrm{NMR}\left(100 \mathrm{MHz}, \mathrm{CDCl}_{3}\right) \delta 38.7,52.5,90.9,115.3,117.4,117.9,122.3,122.6$, 122.6, 126.9, 127.3, 129.2, 129.6, 129.7, 130.8, 130.8, 136.9, 158.3, 166.4, 182.9. IR (KBr) 3350, 2983, 2245, 1676, 1604, 1463, 1197, 929, $707 \mathrm{~cm}^{-1}$. ESI Calcd for $\mathrm{C}_{22} \mathrm{H}_{18} \mathrm{~N}_{2} \mathrm{O}_{3}(\mathrm{M}+\mathrm{Na}) 381.1210$. Found 381.1211. 


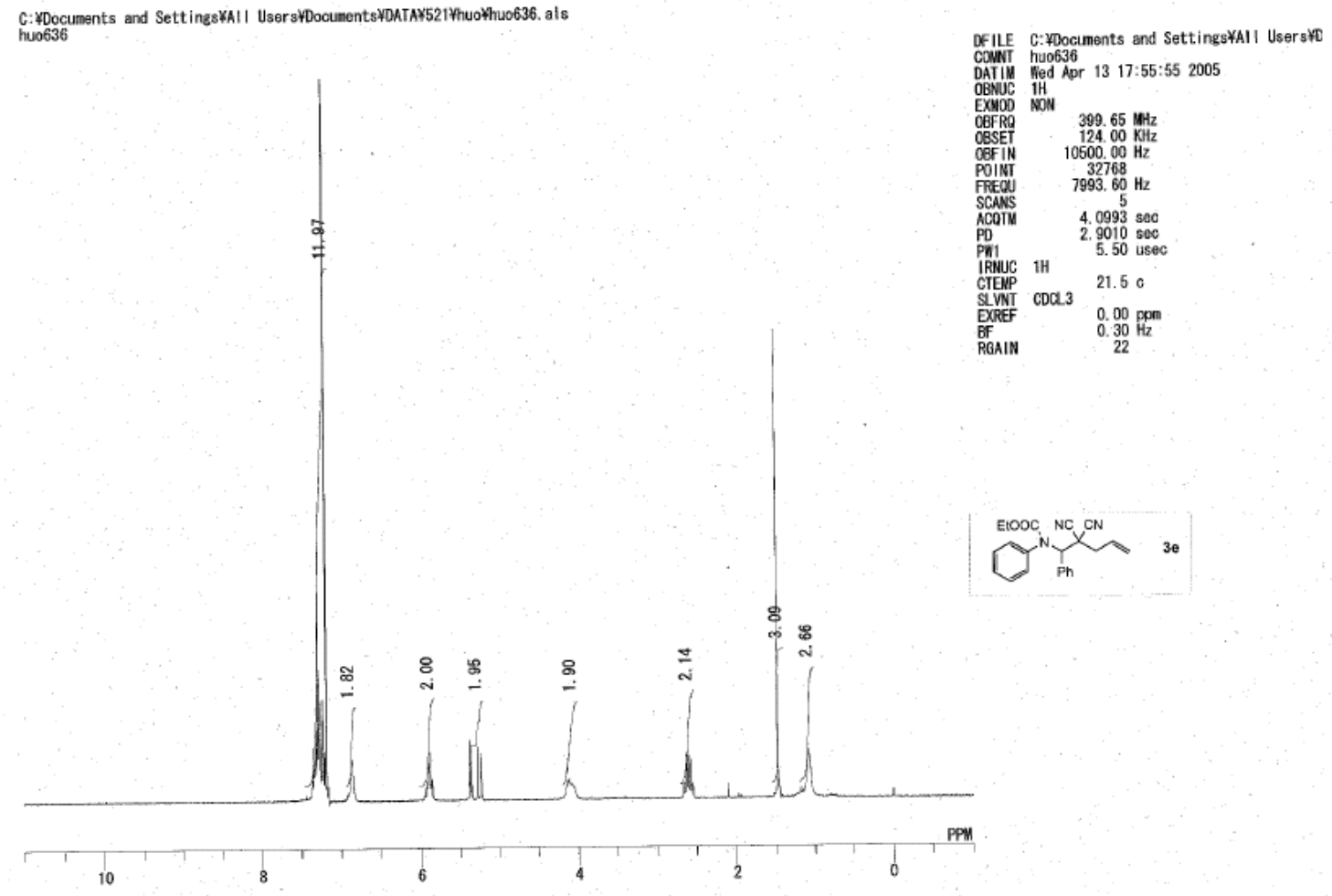

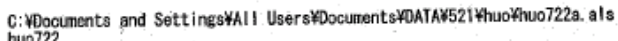

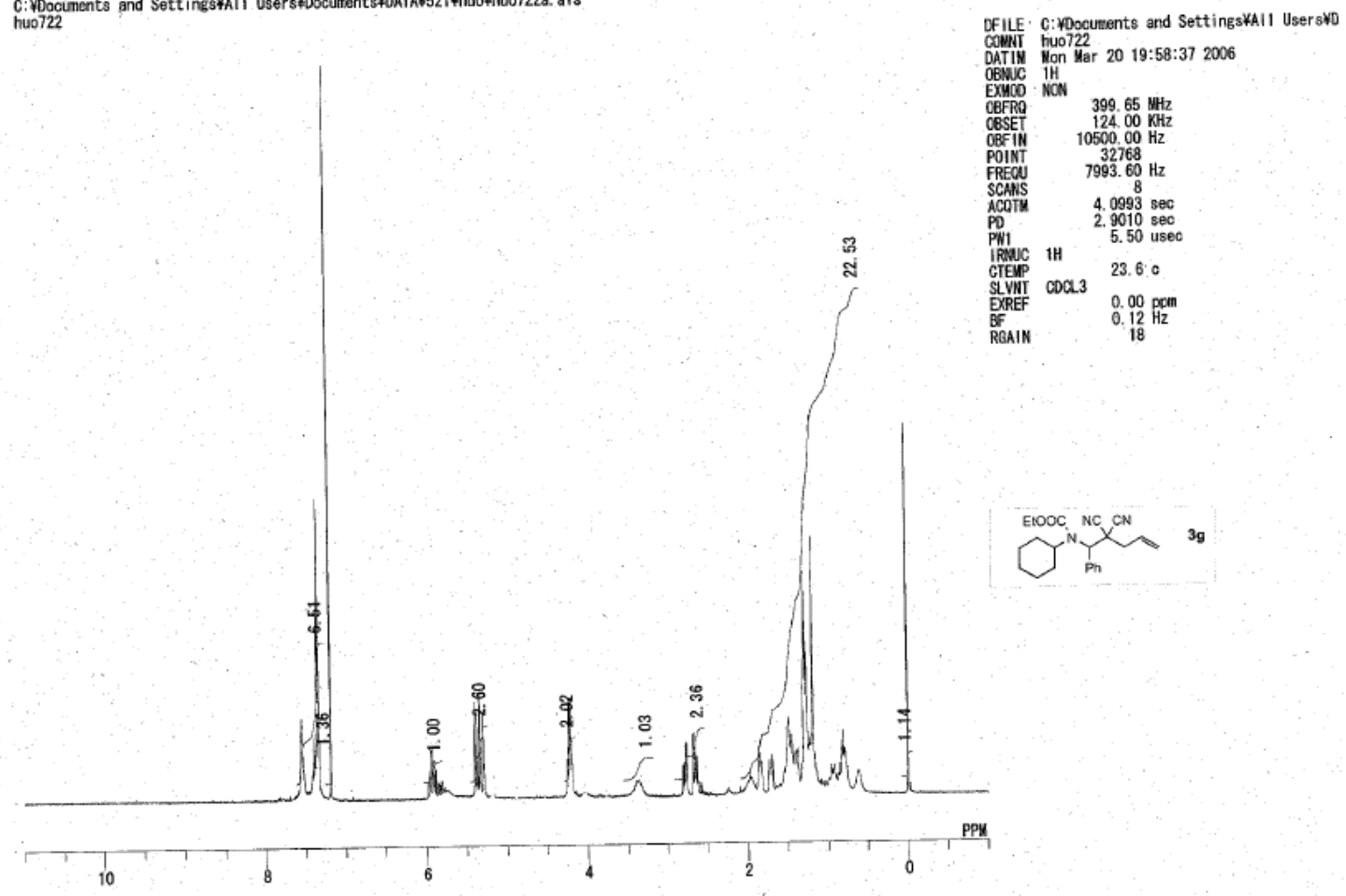




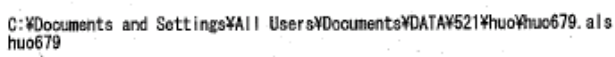

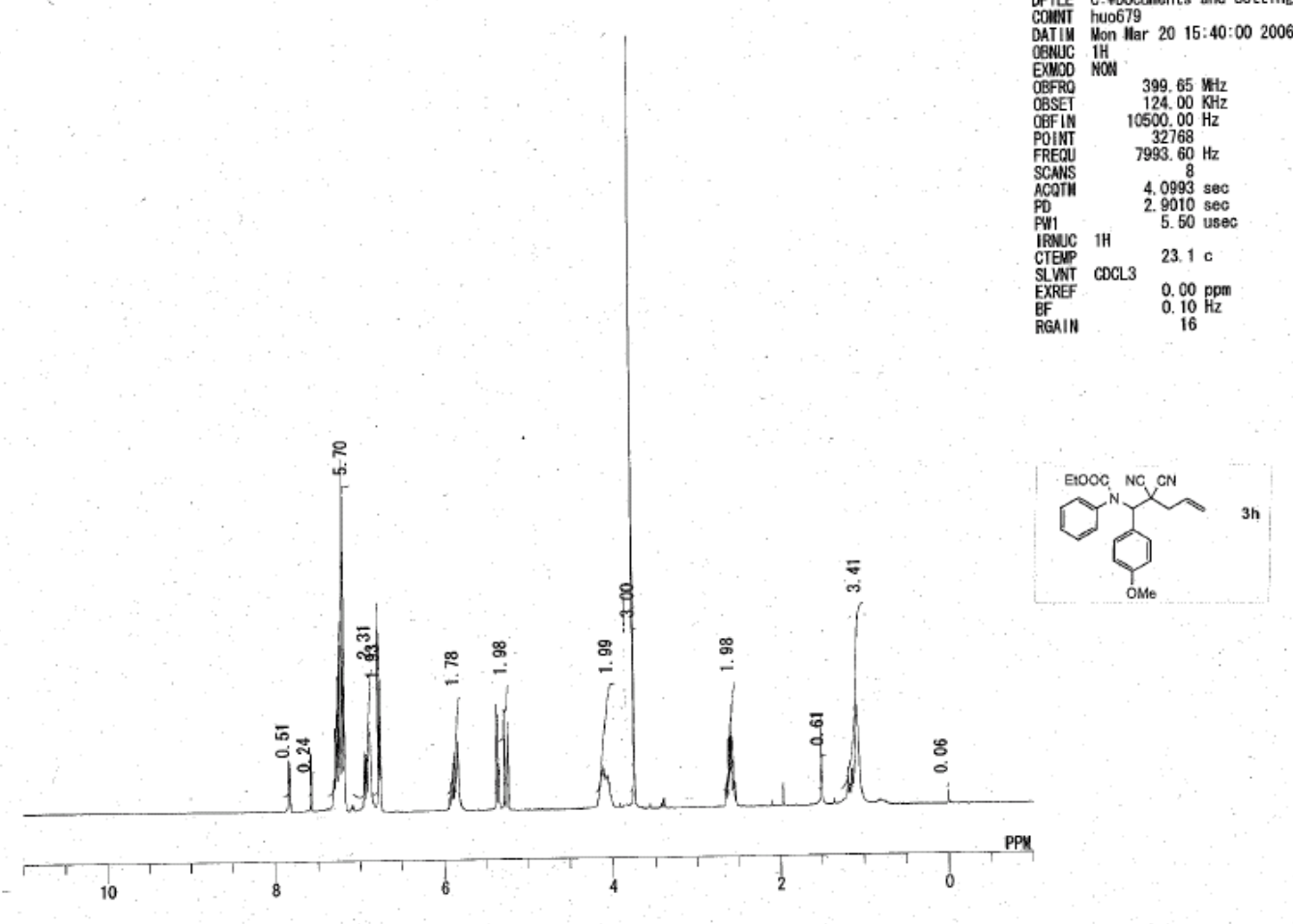

C:FDocuments and SettingsFAlI Users\%Documents\$DATAF521 thuothuo685. als huo685

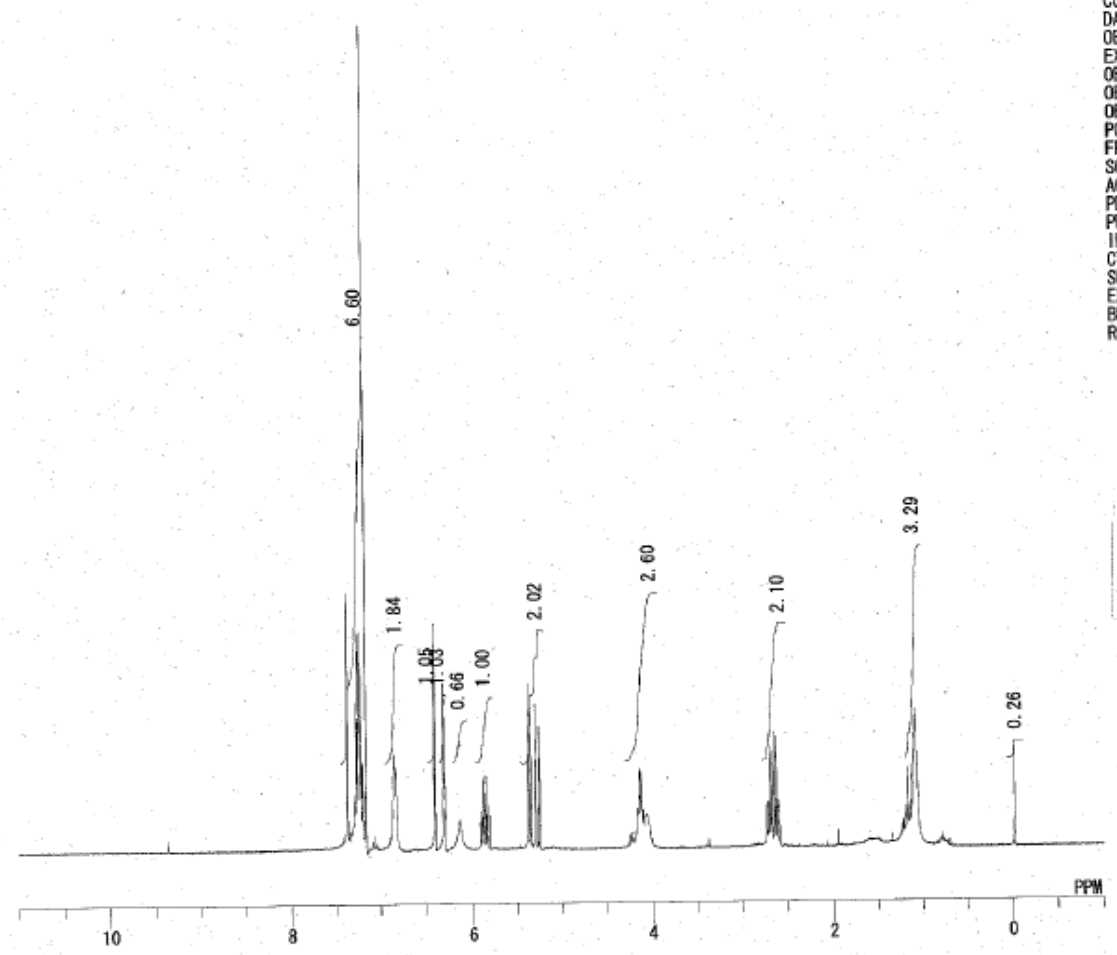

399.65
$\mathrm{YH} 1 \mathrm{Z}$
DFILE C:FDocuments and Settings:All Users $\% 0$

DATIM Hoon Har $20 \quad 15: 40: 002006$

(1)

BFIN $\quad 10500.00 \mathrm{~Hz}$

EQU $\quad 7993.60 \mathrm{~Hz}$

2. $9010 \mathrm{sec}$

1H

23.1

$\quad 0.10 \mathrm{~Hz}$

EtoOC NC CN
FILF C:FDocuments and SettingsłAII Users $F$

Whilu Wo6 20 19:09:40 2006

BNUC IH

BFRQ $399.65 \mathrm{NHz}$

$\quad 124.00 \mathrm{KH}$

100600

FREOU $7993.60 \mathrm{~Hz}$

CCOIY $\quad 4.0993 \mathrm{sec}$

$2.9010 \mathrm{sec}$
$5.50 \mathrm{usec}$

PPVWC $1 \mathrm{H} \quad 23.30$

YNI $\cos 323.30$

$\begin{array}{ll}\text { XXREF } & 0,00 \mathrm{ppa} \\ \text { BF } & 0.12 \mathrm{~Hz} \\ \text { RGAIN } & 19\end{array}$

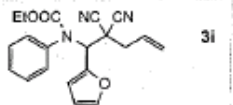




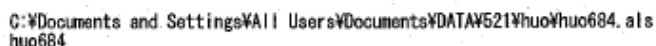

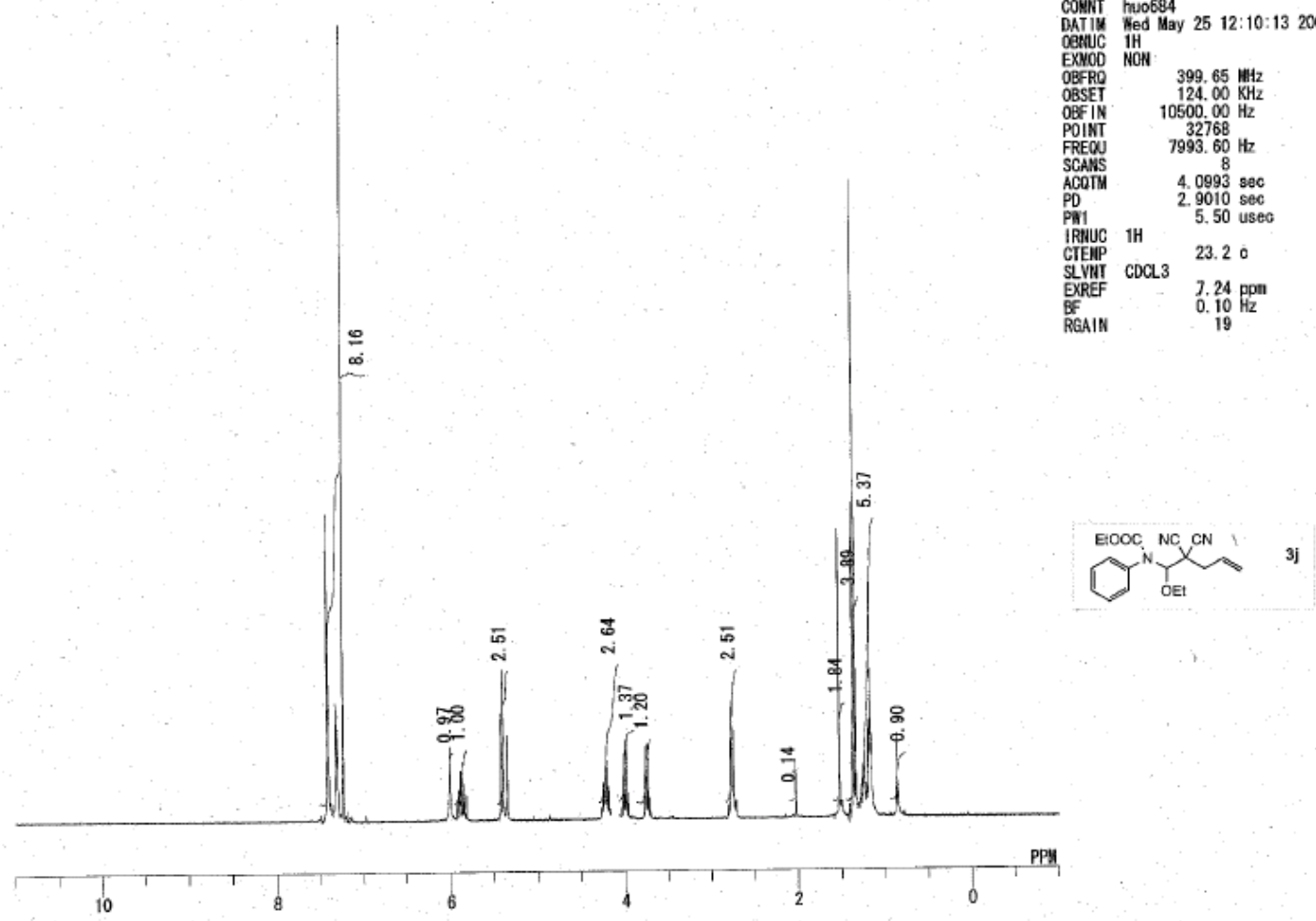

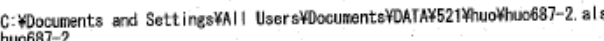

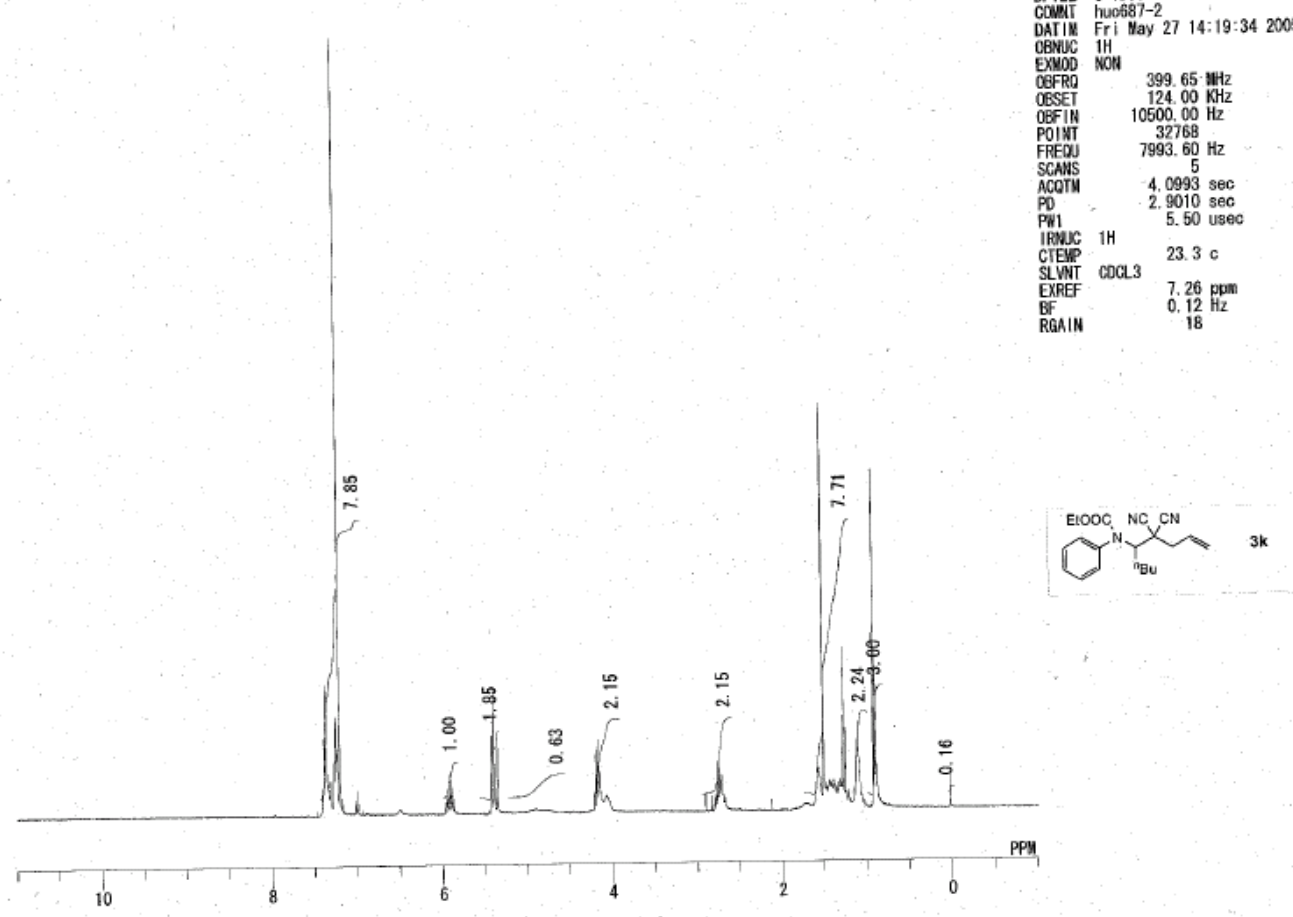




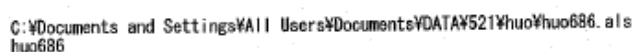

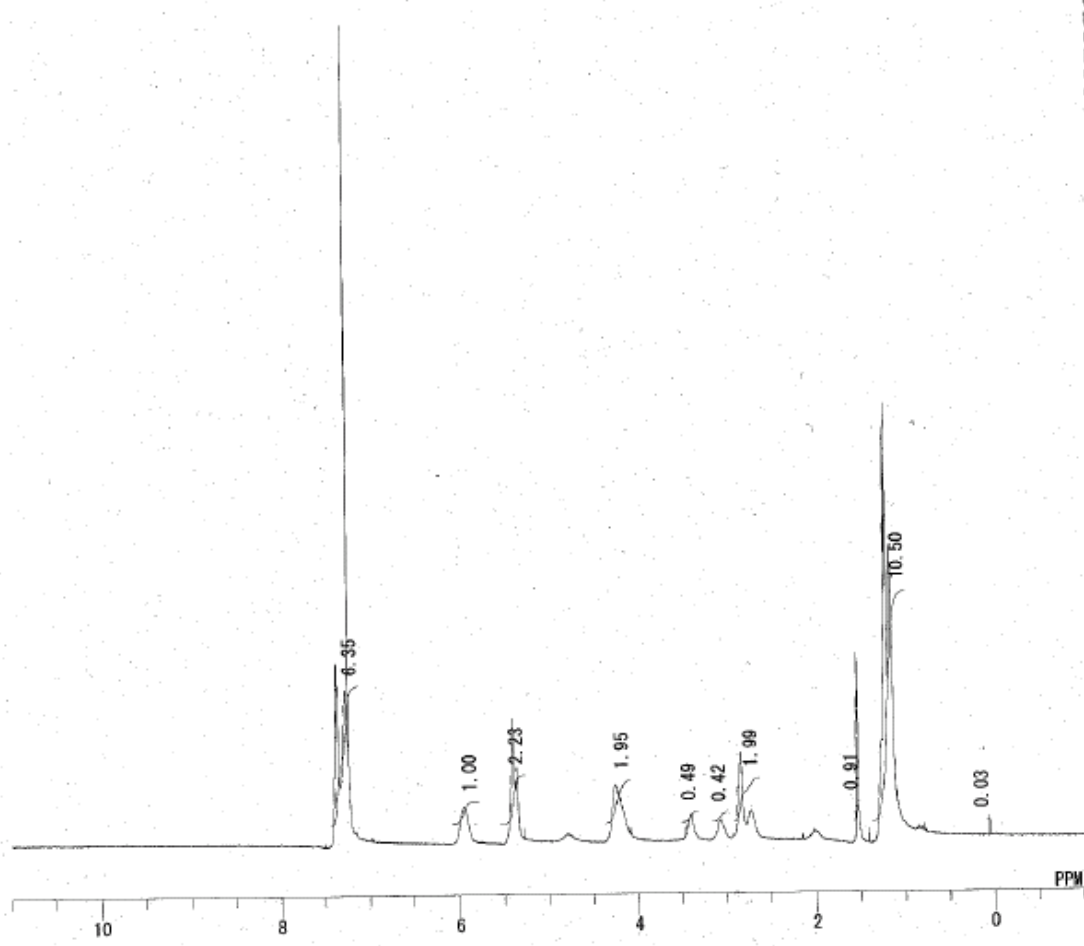

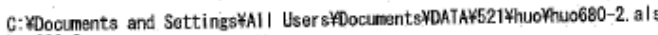
huo680-2

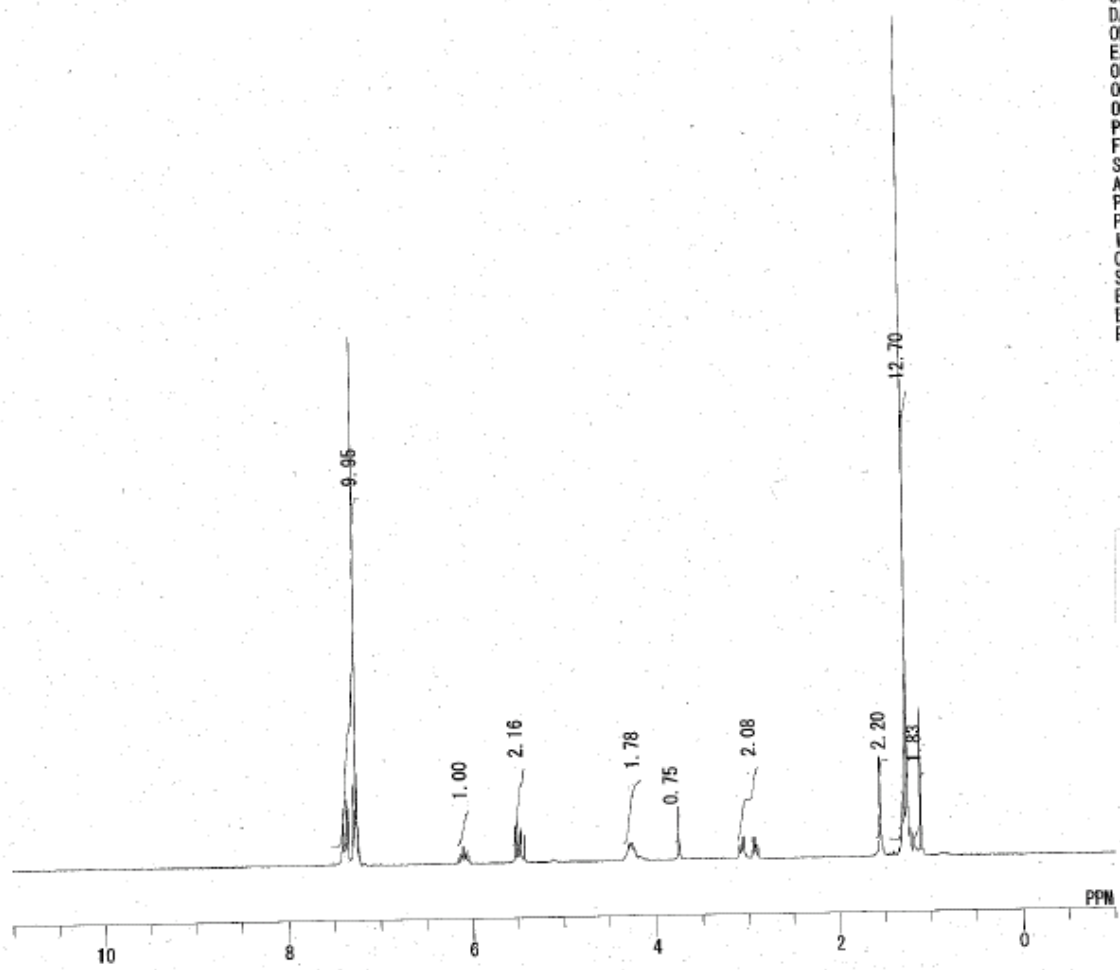

\section{PPy,}

DFILE C: $¥ D$ Documents and Settings $\$ A I I$ User $\$$ ZO

CONAT huo686 27 12:42:31 2005

OENUC $1 \mathrm{H}$

EXNOD NON $399.65 \mathrm{HZ}$
OEFRO

OEF IN $\quad 10500.00 \mathrm{~Hz}$

$\begin{array}{ll}\text { POINT } & 32768 \\ \text { FREQU } & 7993.60 \mathrm{~Hz}\end{array}$

$\begin{array}{ll}\text { ACQTH } & 4.0993 \mathrm{sec} \\ \text { PD } & 2.9010 \mathrm{sec} \\ \text { PW1 } & 5.50 \mathrm{Hsec}\end{array}$

IRANUC $1 \mathrm{H}$

CIBP $23.5 \mathrm{c}$

SLWNT CDCL3

$\begin{array}{ll}7.24 \mathrm{pom} \\ \mathrm{BF} & 0.12 \mathrm{~Hz}\end{array}$

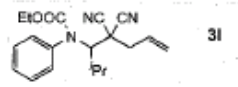



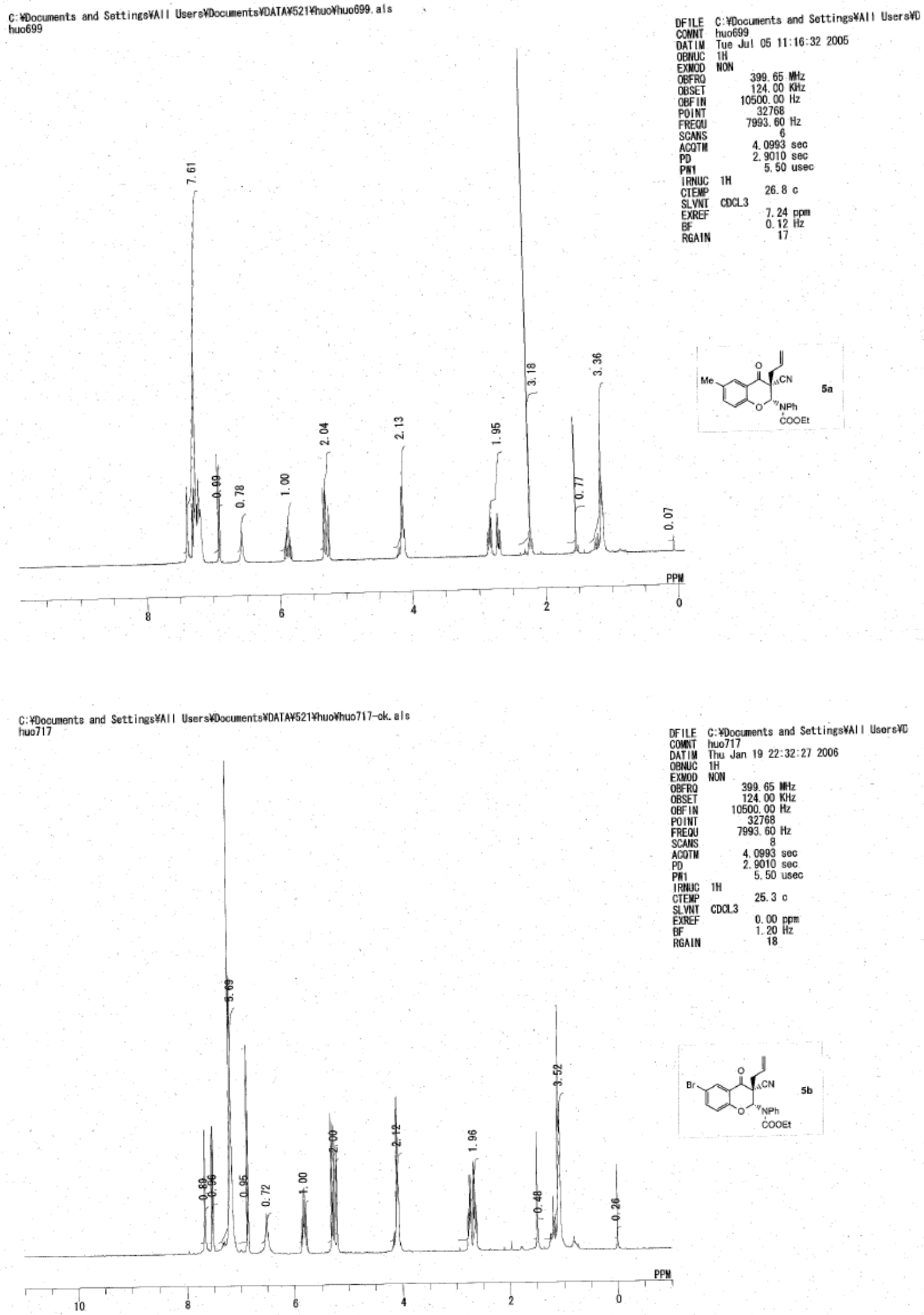


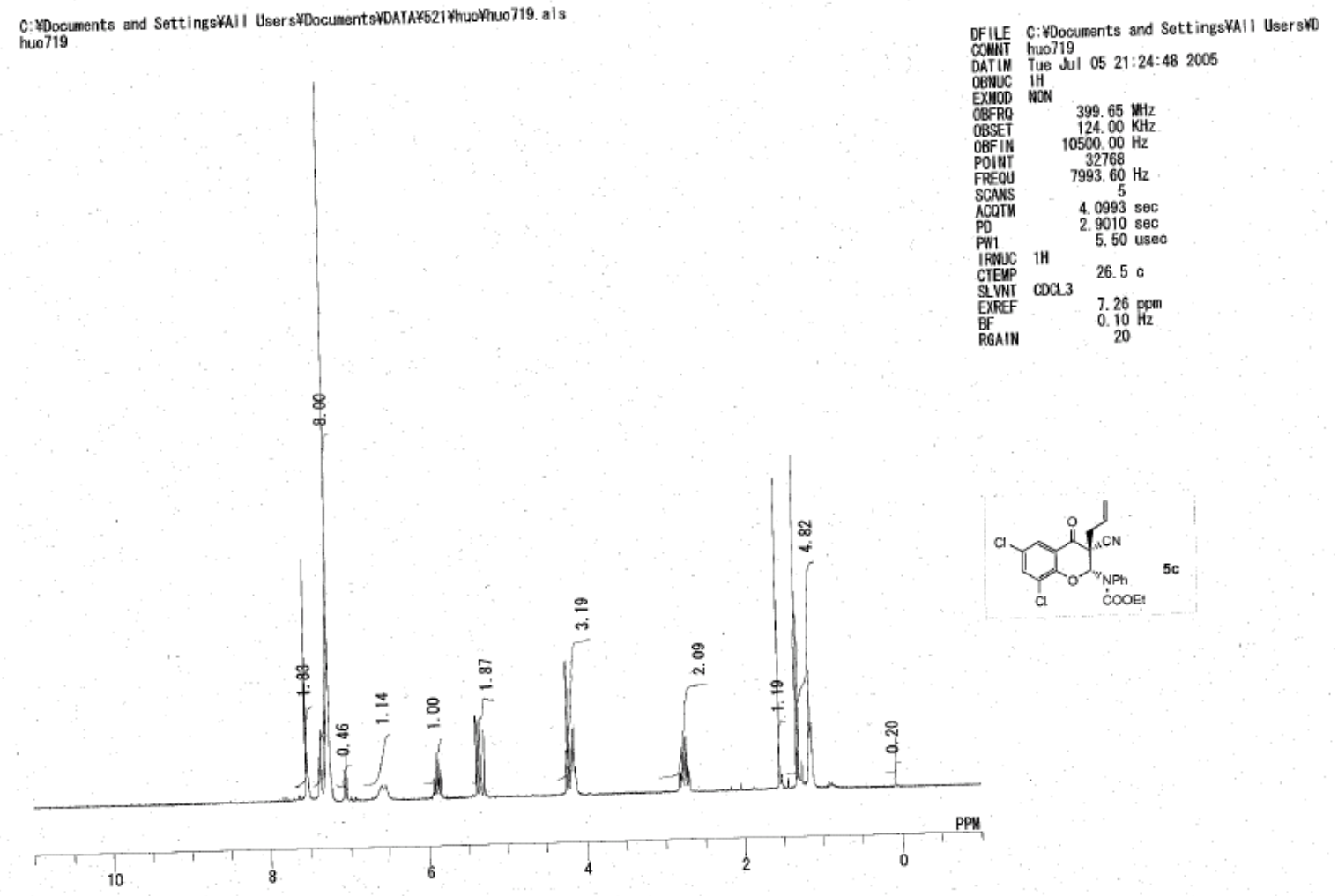

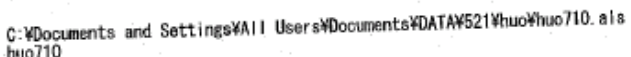

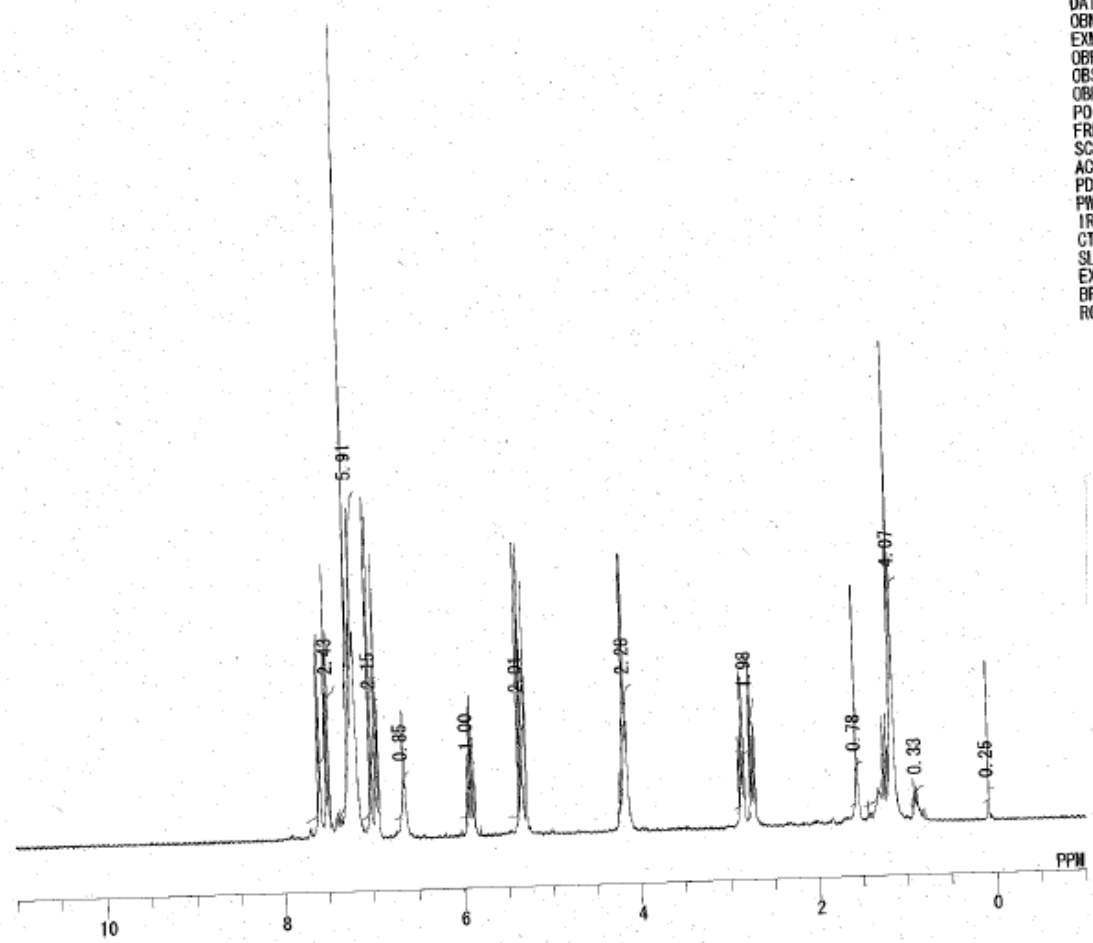

DFILE C:Foounents and Settings $\$$ All User $\$ \$ 0$

CONII huo710 $21: 12: 542005$

OBNUC IH

OBFRO $399.65 \mathrm{WH}$

$\quad 1050000$ H

32768
$\mathrm{H}$

$\quad 4.0993 \mathrm{sec}$

2.90100000
PW1

CTEMP $26.5 \mathrm{G}$

$\begin{array}{ll}\text { SLWNT } & \text { COCL3 } \\ \text { EXREF } & 7.26 \mathrm{pm} \\ \text { BF } & 0.10 \mathrm{HI} \\ \text { PGGIN } & 17\end{array}$

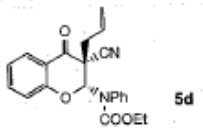




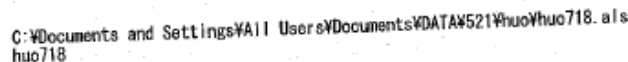

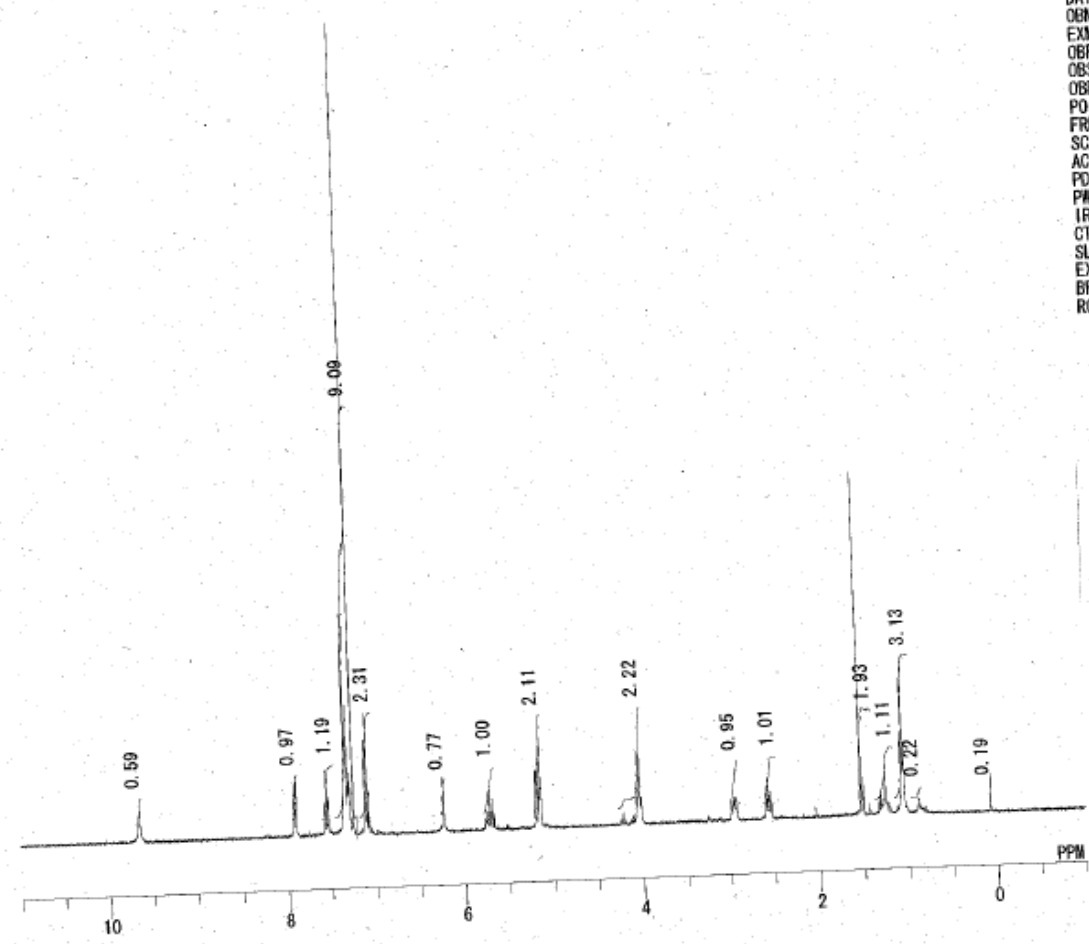

DFILE C:\#Documents and SettingsKAII Usors\%D

COMnT huo718 04 19:55:34 2005

DABUUC

OBFRO NON $399.65 \mathrm{NHz}$

DBFIN $\quad 10500.00 \mathrm{~Hz}$

POIRT $\quad 32768$

FREOU $7993.60 \mathrm{~Hz}$

ACOTY $4.0993 \mathrm{sec}$

$\begin{array}{lr}P D & 2.9010 \mathrm{sec} \\ P H 1 & 5.50 \mathrm{usec}\end{array}$

IRTUC IH 26.6

SLVWT CDCL3 $37.26 \mathrm{ppm}$

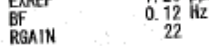

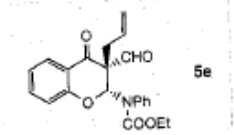

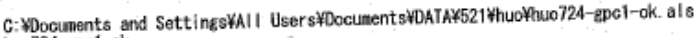
huo 724-gpci-ok

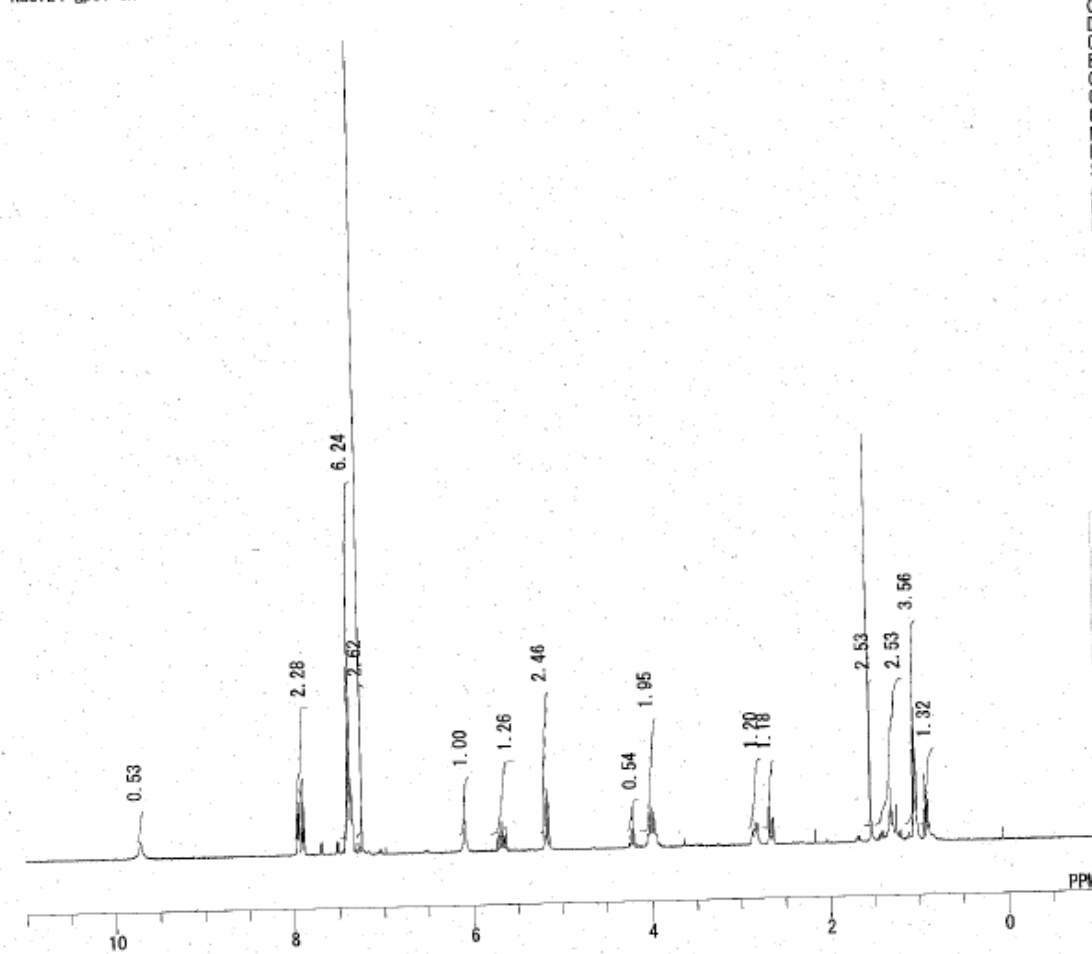

DFILE C: :Documents and Settings*all Users\$C CONWT huo724-goc1-ok
DATIN Fri Feb 03 18:53:04 2006 OBNAC $1 \mathrm{H}$ EXHOD
OBFRO
OBSEI $124.00 \mathrm{KHz}$
OBSEIN : $\quad 10500.00 \mathrm{~Hz}$ POINT
PREOU $\quad \begin{aligned} 32768 \\ \text { PR. }\end{aligned}$ SCANS $\quad 4.0993 \mathrm{sec}$ $\begin{array}{ll}\text { PD } & \text { 2. } 9010 \text { sec } \\ \text { PW1 } & 5.50 \text { usec }\end{array}$ IFPUC $1 \mathrm{H}$ CTEY 25.3 c

$\begin{array}{ll}\text { EXREF } & 0.00 \mathrm{ppm} \\ \text { BF } & 0.12 \mathrm{~Hz} \\ \text { BGAIN } & 22\end{array}$

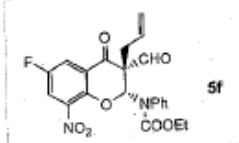


C:FDocunents and SettingsłAlI UsersłDoouments4DATAF521 thuo*huo727. als huo727

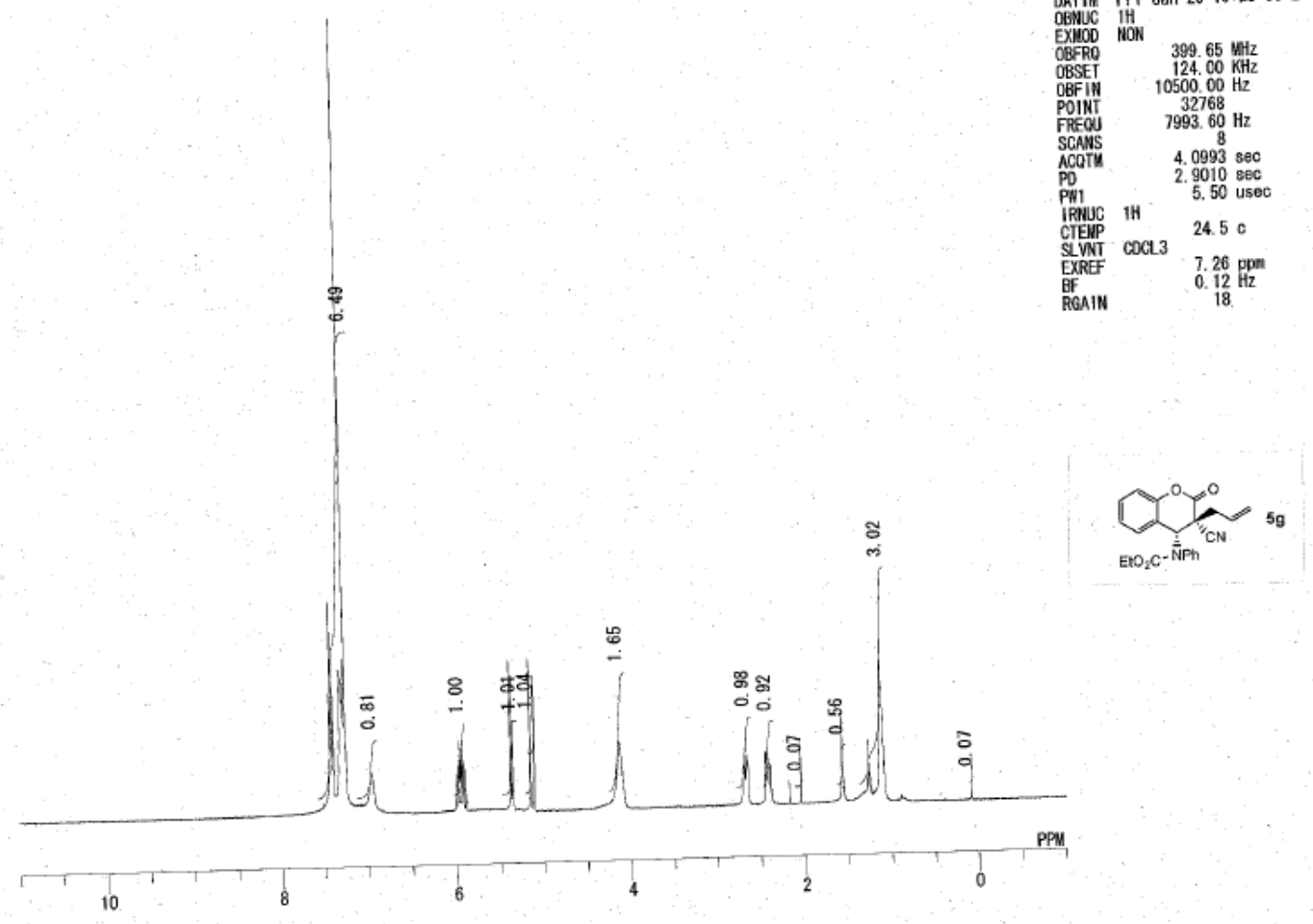

C: ¥0ocuments and Settings\$AII Users\$Documents\$DATAF521 thuo\%huo707-crude. als C: \#poouments
huo707-crude

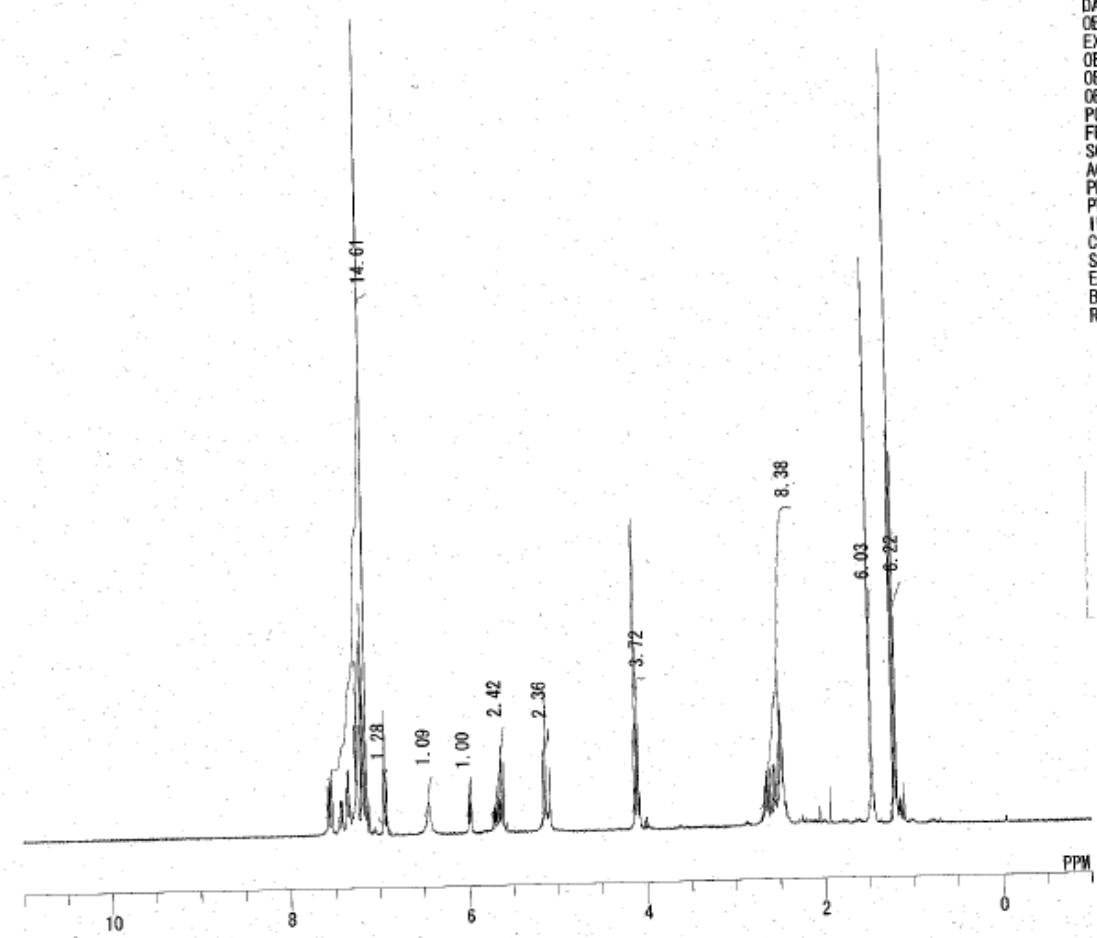

SLVNT COCL3 7.26 pp$$
\text { . }
$$

DFILE C: FDocunents and Settings $¥ A 11$ Users\$D COMNI huo727 DATIN Fri 32768

4. $0993 \mathrm{sec}$ 5.50 usec

WN1 14 .

CIEAPP 24.5

$\begin{array}{ll}\mathrm{EF} & 0.12 \mathrm{~Hz} \\ \mathrm{RGAIN} & 18\end{array}$ 

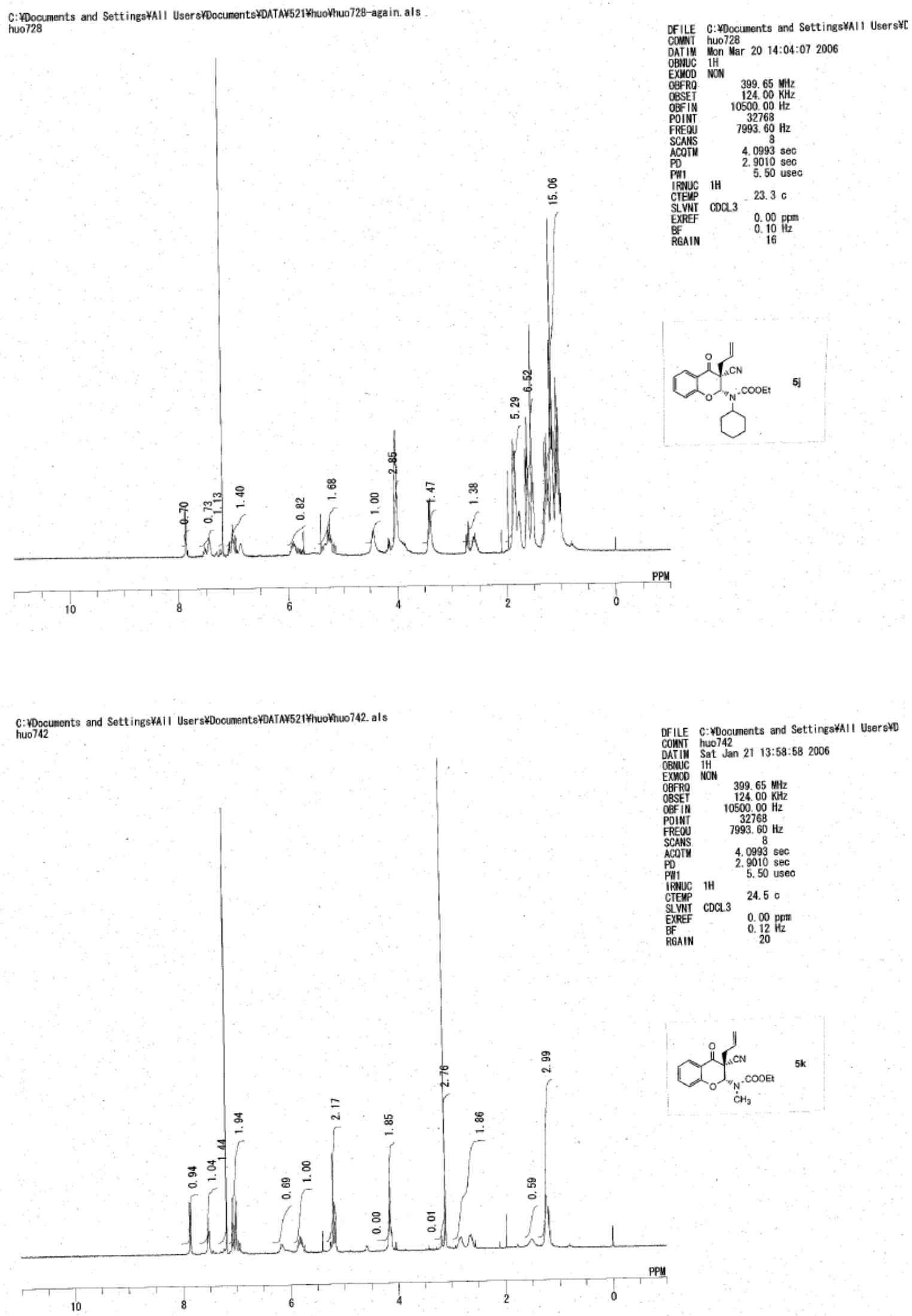


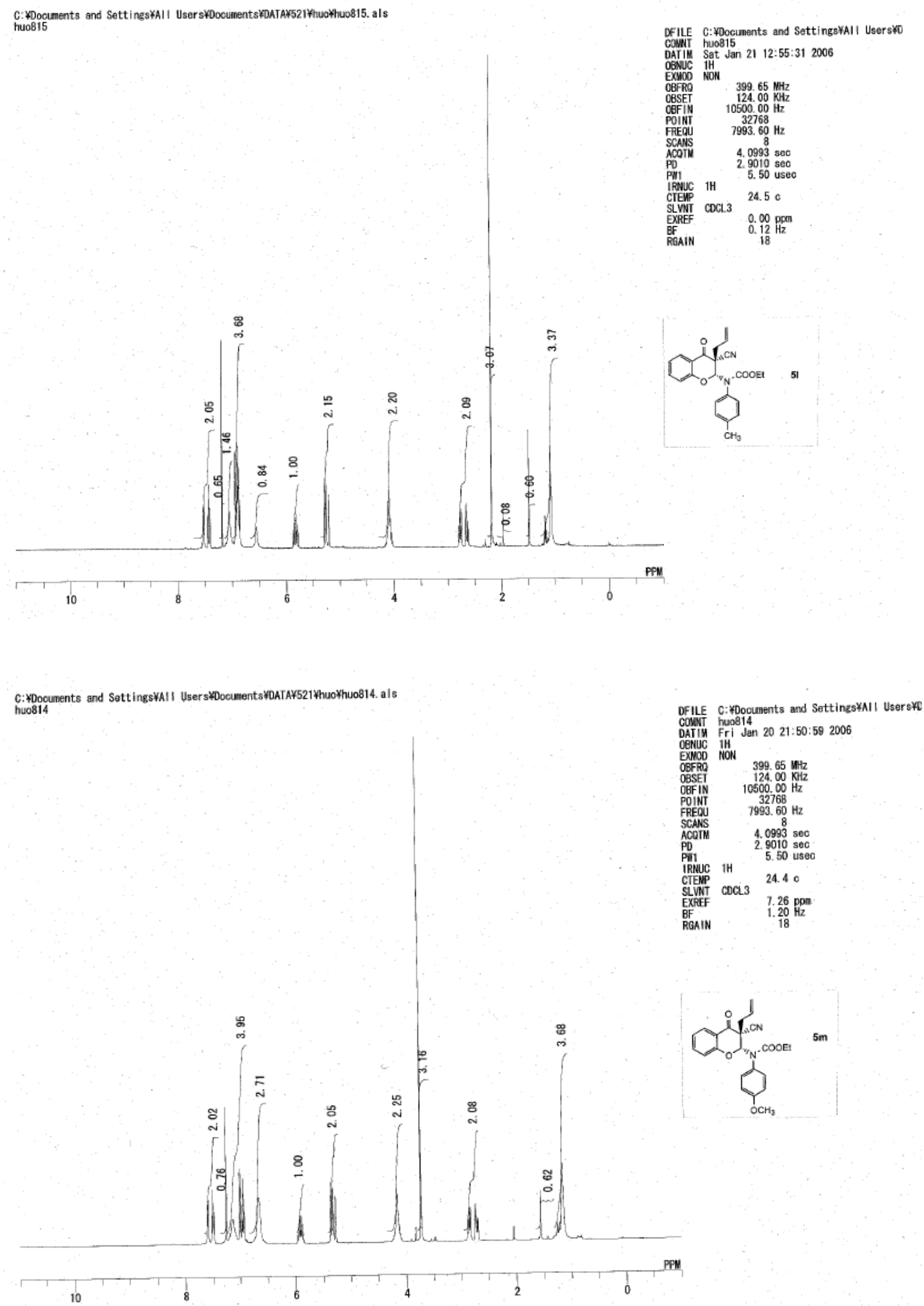


C:FDocuments and Settings:AII Users:Documents:DATAF521 thuołhuo778. als

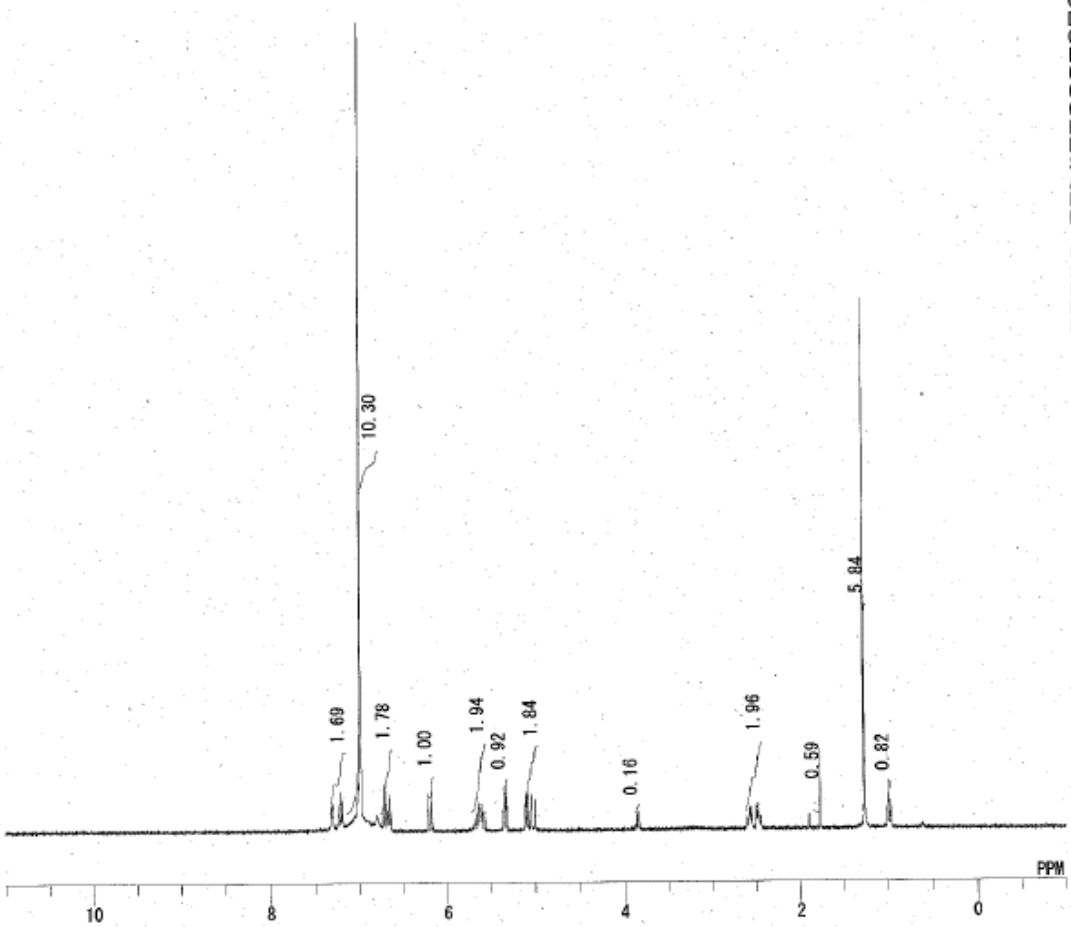

DFILE C:FDocuments and Settings:AII Users¥D

18:08:302006

EXWNCD NON

399. 65 . MHE

$\quad 124.00 \mathrm{KH}$

$10500,00 \mathrm{H}$
32768

RREOU $\quad 7993.60 \mathrm{~Hz}$

CQTM $\quad 4.0993 \mathrm{se}$

2. $9010 \mathrm{sec}$

NUC 1H 24.70

CDCL3 $24.7 \mathrm{C}$

$\begin{array}{ll}\text { EXRE } & 0.00 \mathrm{pom} \\ \mathrm{BF} & 0.12 \mathrm{~Hz}\end{array}$

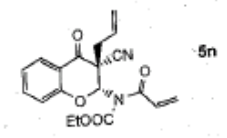


X-Ray Structure of $\mathbf{5 n}$

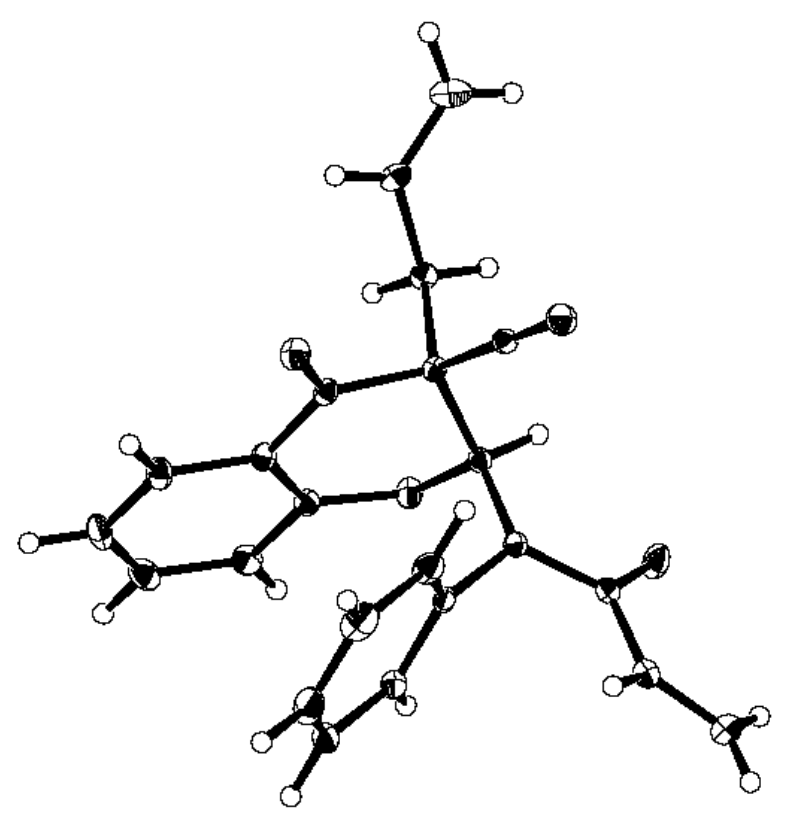

Experimental Details

A. Crystal Data

Empirical Formula

$\mathrm{C}_{22} \mathrm{H}_{18} \mathrm{~N}_{2} \mathrm{O}_{3}$

Formula Weight

358.40

Crystal Color, Habit

colorless, prism

Crystal Dimensions

$0.20 \times 0.20 \times 0.20 \mathrm{~mm}$

Crystal System

orthorhombic

Lattice Type

Primitive 
Detector Position

Pixel Size

Lattice Parameters

Space Group

$\mathrm{Z}$ value

$\mathrm{D}_{\text {calc }}$

$\mathrm{F}_{000}$

$\mu(\operatorname{MoK} \alpha)$
$50.96 \mathrm{~mm}$

$0.137 \mathrm{~mm}$

$$
\begin{aligned}
& \mathrm{a}=15.706(5) \AA \\
& \mathrm{b}=8.930(3) \AA \\
& \mathrm{c}=12.969(5) \AA \\
& \mathrm{V}=1818.8(11) \AA^{3}
\end{aligned}
$$

$\operatorname{Pna}_{1}(\# 33)$

4

$1.309 \mathrm{~g} / \mathrm{cm}^{3}$

752.00

$0.881 \mathrm{~cm}^{-1}$

\section{B. Intensity Measurements}

Detector

Goniometer

Radiation

Detector Aperture

Data Images

$\omega$ oscillation Range $(\chi=45.0, \phi=0.0)$

Exposure Rate

Detector Swing Angle
Rigaku Saturn

Rigaku AFC10

$\operatorname{MoK} \alpha(\lambda=0.71070 \AA)$

graphite monochromated

$70 \mathrm{~mm} \times 70 \mathrm{~mm}$

1800 exposures

$-110.0-70.0^{\circ}$

$40.0 \mathrm{sec} . / 0$

$-19.65^{\circ}$ 


\begin{tabular}{|c|c|}
\hline$\omega$ oscillation Range $(\chi=45.0, \phi=90.0)$ & $-110.0-70.0^{\circ}$ \\
\hline Exposure Rate & 40.0 sec. $/ 0$ \\
\hline Detector Swing Angle & $-19.65^{0}$ \\
\hline$\omega$ oscillation Range $(\chi=45.0, \phi=180.0)$ & $-110.0-70.0^{\circ}$ \\
\hline Exposure Rate & $40.0 \mathrm{sec} . / 0$ \\
\hline Detector Swing Angle & $-19.65^{\circ}$ \\
\hline$\omega$ oscillation Range $(\chi=45.0, \phi=270.0)$ & $-110.0-70.0^{0}$ \\
\hline Exposure Rate & 40.0 sec. $/ 0$ \\
\hline Detector Swing Angle & $-19.65^{0}$ \\
\hline Detector Position & $50.96 \mathrm{~mm}$ \\
\hline Pixel Size & $0.137 \mathrm{~mm}$ \\
\hline $2 \theta_{\max }$ & 54.90 \\
\hline
\end{tabular}

No. of Reflections Measured

Total: 23884

Unique: $2026\left(\mathrm{R}_{\text {int }}=0.045\right)$

Corrections

Lorentz-polarization

Absorption

(trans. factors: $0.836-0.983$ )

C. Structure Solution and Refinement

Structure Solution

Direct Methods (SIR97) 
Refinement

Function Minimized

Least Squares Weights

$2 \theta_{\max }$ cutoff

Anomalous Dispersion

No. Observations (All reflections)

No. Variables

Reflection/Parameter Ratio

Residuals: R1 (I>2.00б(I))

Residuals: R (All reflections)

Residuals: wR2 (All reflections)

Goodness of Fit Indicator

Max Shift/Error in Final Cycle

Maximum peak in Final Diff. Map

Minimum peak in Final Diff. Map
Full-matrix least-squares on $\mathrm{F}^{2}$

$\Sigma_{\mathrm{w}}\left(\mathrm{Fo}_{\mathrm{o}}^{2}-\mathrm{Fc}^{2}\right)^{2}$

$1 /\left[1.0000 \sigma\left(\mathrm{Fo}^{2}\right)\right] /\left(4 \mathrm{Fo}_{0}{ }^{2}\right)$

$54.9^{\circ}$

All non-hydrogen atoms

2026

262

7.73

0.0264

0.0297

0.0595

0.994

0.000

$0.17 \mathrm{e}^{-} / \AA^{3}$

$-0.16 \mathrm{e}^{-} / \AA^{3}$

Table 1. Atomic coordinates and $\mathrm{B}_{\mathrm{iso}} / \mathrm{B}_{\mathrm{eq}}$

$\begin{array}{lllll}\text { atom } & \mathrm{x} & \mathrm{y} & \mathrm{z} & \mathrm{B}_{\mathrm{eq}} \\ \mathrm{O}(1) & 0.97423(8) & -0.02305(12) & 0.31643(9) & 2.06(2) \\ \mathrm{O}(2) & 0.97875(6) & 0.28712(12) & 0.08122(9) & 1.80(2) \\ \mathrm{O}(3) & 0.83751(8) & 0.19045(14) & -0.10286(9) & 2.44(2)\end{array}$




\begin{tabular}{|c|c|c|c|c|}
\hline $\mathrm{N}(1)$ & $0.84498(8)$ & $0.15837(13)$ & $0.07136(10)$ & $1.53(2)$ \\
\hline $\mathrm{N}(2)$ & $0.89265(10)$ & $-0.22605(16)$ & $0.10636(12)$ & $2.33(3)$ \\
\hline $\mathrm{C}(1)$ & $0.97710(10)$ & $0.02367(17)$ & $0.13307(12)$ & $1.39(2)$ \\
\hline $\mathrm{C}(2)$ & $0.97950(10)$ & $0.06919(19)$ & $0.24791(12)$ & $1.49(3)$ \\
\hline C(3) & $0.99492(12)$ & $0.22972(18)$ & $0.26454(12)$ & $1.59(3)$ \\
\hline C(4) & $1.01022(12)$ & $0.2852(2)$ & $0.36419(12)$ & $2.02(3)$ \\
\hline$C(5)$ & $1.02715(12)$ & $0.4355(2)$ & $0.37964(13)$ & $2.45(4)$ \\
\hline $\mathrm{C}(6)$ & $1.02902(12)$ & $0.5317(2)$ & $0.29542(14)$ & $2.43(3)$ \\
\hline $\mathrm{C}(7)$ & $1.01350(12)$ & $0.48045(19)$ & $0.19671(13)$ & $2.03(3)$ \\
\hline $\mathrm{C}(8)$ & $0.99517(11)$ & $0.32975(18)$ & $0.18160(12)$ & $1.62(3)$ \\
\hline C(9) & $0.93702(10)$ & $0.14647(16)$ & $0.06403(12)$ & $1.46(2)$ \\
\hline$C(10)$ & $0.80526(10)$ & $0.19647(19)$ & $0.16891(12)$ & $1.67(3)$ \\
\hline $\mathrm{C}(11)$ & $0.78083(11)$ & $0.0841(2)$ & $0.23685(13)$ & $2.29(3)$ \\
\hline $\mathrm{C}(12)$ & $0.74298(13)$ & $0.1232(2)$ & $0.32975(14)$ & $3.25(4)$ \\
\hline C(13) & $0.72899(12)$ & $0.2712(2)$ & $0.35375(16)$ & $3.56(5)$ \\
\hline $\mathrm{C}(14)$ & $0.75243(13)$ & $0.3827(2)$ & $0.28555(14)$ & $3.10(4)$ \\
\hline$C(15)$ & $0.79091(12)$ & $0.3457(2)$ & $0.19231(13)$ & $2.25(3)$ \\
\hline$C(16)$ & $1.07154(10)$ & $0.00107(18)$ & $0.09708(12)$ & $1.64(3)$ \\
\hline $\mathrm{C}(17)$ & $1.11514(11)$ & $-0.13015(19)$ & $0.14591(13)$ & $1.98(3)$ \\
\hline C(18) & $1.14987(12)$ & $-0.2399(2)$ & $0.09273(17)$ & $3.01(4)$ \\
\hline C(19) & $0.92929(10)$ & $-0.11722(17)$ & $0.11937(12)$ & $1.56(3)$ \\
\hline$C(20)$ & $0.80055(11)$ & $0.18061(18)$ & $-0.02029(12)$ & $1.66(3)$ \\
\hline $\mathrm{C}(21)$ & $0.70599(12)$ & $0.1853(2)$ & $-0.01235(13)$ & $2.07(3)$ \\
\hline $\mathrm{C}(22)$ & $0.65884(13)$ & $0.2226(2)$ & $-0.09241(16)$ & $2.79(4)$ \\
\hline
\end{tabular}

Table 2. Atomic coordinates and $B$ iso involving hydrogens/Beq

$\begin{array}{lcccc}\text { atom } & \mathrm{x} & \mathrm{y} & \mathrm{z} & \mathrm{B}_{\mathrm{eq}} \\ \mathrm{H}(1) & 1.0084 & 0.2190 & 0.4212 & 2.43 \\ \mathrm{H}(2) & 1.0379 & 0.4734 & 0.4469 & 2.94 \\ \mathrm{H}(3) & 1.0414 & 0.6344 & 0.3063 & 2.91 \\ \mathrm{H}(4) & 1.0148 & 0.5475 & 0.1400 & 2.41 \\ \mathrm{H}(5) & 0.9493 & 0.1186 & -0.0046 & 1.72 \\ \mathrm{H}(6) & 0.7900 & -0.0181 & 0.2194 & 2.74\end{array}$




$\begin{array}{lllll}\mathrm{H}(7) & 0.7269 & 0.0477 & 0.3772 & 3.88 \\ \mathrm{H}(8) & 0.7034 & 0.2976 & 0.4175 & 4.28 \\ \mathrm{H}(9) & 0.7420 & 0.4845 & 0.3023 & 3.70 \\ \mathrm{H}(10) & 0.8074 & 0.4207 & 0.1450 & 2.70 \\ \mathrm{H}(11) & 1.1023 & 0.0889 & 0.1141 & 1.96 \\ \mathrm{H}(12) & 1.0713 & -0.0128 & 0.0247 & 1.98 \\ \mathrm{H}(13) & 1.1175 & -0.1340 & 0.2186 & 2.38 \\ \mathrm{H}(14) & 1.1770 & -0.3199 & 0.1277 & 3.61 \\ \mathrm{H}(15) & 1.1486 & -0.2382 & 0.0193 & 3.64 \\ \mathrm{H}(16) & 0.6794 & 0.1604 & 0.0511 & 2.48 \\ \mathrm{H}(17) & 0.5989 & 0.2256 & -0.0869 & 3.34 \\ \mathrm{H}(18) & 0.6860 & 0.2474 & -0.1558 & 3.36 \\ & & & & \\ \left.\mathrm{~B}_{\mathrm{eq}}=8 / 3 \pi^{2}\left(\mathrm{U}_{11}\left(\mathrm{aa}^{*}\right)^{2}+\mathrm{U}_{22}\left(\mathrm{bb}^{*}\right)^{2}+\mathrm{U}_{33}\left(\mathrm{cc}^{*}\right)^{2}+2 \mathrm{U}_{12}\left(\mathrm{aa}^{*} \mathrm{bb} b^{*}\right) \cos \gamma+2 \mathrm{U}_{13}\left(\mathrm{aa}^{*} \mathrm{cc}\right)^{*}\right) \cos \beta+2 \mathrm{U}_{23}\left(\mathrm{bb}^{*} \mathrm{cc}\right) \cos \alpha\right)\end{array}$


Table 3. Anisotropic displacement parameters

$\begin{array}{lllllll}\text { atom } & \mathrm{U}_{11} & \mathrm{U}_{22} & \mathrm{U} 33 & \mathrm{U}_{12} & \mathrm{U}_{13} & \mathrm{U}_{23} \\ \mathrm{O}(1) & 0.0317(6) & 0.0266(6) & 0.0199(5) & -0.0021(5) & 0.0006(5) & 0.0068(5) \\ \mathrm{O}(2) & 0.0303(6) & 0.0190(5) & 0.0190(5) & -0.0041(4) & -0.0027(5) & 0.0035(4) \\ \mathrm{O}(3) & 0.0296(7) & 0.0455(7) & 0.0177(6) & 0.0003(5) & -0.0030(5) & 0.0013(5) \\ \mathrm{N}(1) & 0.0205(6) & 0.0225(6) & 0.0152(6) & 0.0032(5) & -0.0007(5) & -0.0010(5) \\ \mathrm{N}(2) & 0.0276(7) & 0.0259(7) & 0.0350(9) & -0.0029(6) & -0.0011(6) & -0.0029(6) \\ \mathrm{C}(1) & 0.0178(7) & 0.0176(7) & 0.0175(7) & -0.0012(5) & -0.0010(6) & 0.0014(5) \\ \mathrm{C}(2) & 0.0177(8) & 0.0245(8) & 0.0146(7) & 0.0001(6) & 0.0003(5) & 0.0015(6) \\ \mathrm{C}(3) & 0.0207(8) & 0.0217(8) & 0.0179(7) & -0.0021(6) & -0.0007(6) & 0.0011(6) \\ \mathrm{C}(4) & 0.0276(9) & 0.0319(9) & 0.0174(7) & -0.0022(7) & 0.0015(7) & -0.0014(7) \\ \mathrm{C}(5) & 0.0333(10) & 0.0347(10) & 0.0251(9) & -0.0054(8) & 0.0004(7) & -0.0116(7) \\ \mathrm{C}(6) & 0.0308(9) & 0.0229(9) & 0.0386(11) & -0.0048(7) & 0.0018(8) & -0.0081(7) \\ \mathrm{C}(7) & 0.0276(9) & 0.0218(8) & 0.0279(9) & -0.0027(7) & -0.0001(7) & 0.0018(7) \\ \mathrm{C}(8) & 0.0192(7) & 0.0221(8) & 0.0205(8) & 0.0001(6) & -0.0005(7) & -0.0012(6) \\ \mathrm{C}(9) & 0.0212(7) & 0.0186(7) & 0.0156(7) & 0.0001(5) & 0.0003(6) & -0.0006(5) \\ \mathrm{C}(10) & 0.0159(7) & 0.0321(9) & 0.0156(7) & 0.0032(6) & -0.0020(6) & -0.0048(6) \\ \mathrm{C}(11) & 0.0217(8) & 0.0391(10) & 0.0261(9) & 0.0010(7) & 0.0015(7) & 0.0025(7) \\ \mathrm{C}(12) & 0.0264(9) & 0.0690(14) & 0.0279(10) & 0.0035(10) & 0.0065(8) & 0.0070(9) \\ \mathrm{C}(13) & 0.0277(11) & 0.0855(17) & 0.0222(9) & 0.0143(10) & 0.0027(8) & -0.0153(10) \\ \mathrm{C}(14) & 0.0290(9) & 0.0550(12) & 0.0339(9) & 0.0134(10) & -0.0073(9) & -0.0232(9) \\ \mathrm{C}(15) & 0.0250(8) & 0.0355(10) & 0.0249(9) & 0.0062(7) & -0.0048(7) & -0.0087(7) \\ \mathrm{C}(16) & 0.0187(7) & 0.0234(8) & 0.0201(7) & -0.0015(6) & 0.0024(6) & 0.0010(6) \\ \mathrm{C}(17) & 0.0182(8) & 0.0297(8) & 0.0274(9) & 0.0007(6) & -0.0008(6) & 0.0047(7) \\ \mathrm{C}(18) & 0.0320(10) & 0.0356(10) & 0.0469(12) & 0.0105(8) & 0.0045(9) & 0.0069(9) \\ \mathrm{C}(19) & 0.0195(8) & 0.0222(8) & 0.0175(7) & 0.0027(6) & 0.0014(6) & -0.0007(6) \\ \mathrm{C}(20) & 0.0244(9) & 0.0177(7) & 0.0211(8) & 0.0015(6) & -0.0026(7) & -0.0033(6) \\ \mathrm{C}(21) & 0.0276(9) & 0.0278(9) & 0.0233(8) & 0.0029(7) & -0.0014(7) & -0.0060(7) \\ \mathrm{C}(22) & 0.0319(10) & 0.0376(10) & 0.0364(10) & 0.0079(8) & -0.0097(8) & -0.0060(8)\end{array}$

The general temperature factor expression: $\exp \left(-2 \pi^{2}\left(\mathrm{a}^{* 2} \mathrm{U}_{11} \mathrm{~h}^{2}+\mathrm{b}^{* 2} \mathrm{U}_{22} \mathrm{k}^{2}+\mathrm{c}^{* 2} \mathrm{U}_{33^{2}}{ }^{2}\right.\right.$ $\left.\left.+2 a * b * U_{12} h k+2 a * c * U_{13} h l+2 b * c * U_{23} k l\right)\right)$ 
Table 4. Bond lengths (̊)

$\begin{array}{llllll}\text { atom } & \text { atom } & \text { distance } & \text { atom } & \text { atom } & \text { distance } \\ \mathrm{O}(1) & \mathrm{C}(2) & 1.2145(19) & \mathrm{O}(2) & \mathrm{C}(8) & 1.3806(19) \\ \mathrm{O}(2) & \mathrm{C}(9) & 1.4341(18) & \mathrm{O}(3) & \mathrm{C}(20) & 1.221(2) \\ \mathrm{N}(1) & \mathrm{C}(9) & 1.453(2) & \mathrm{N}(1) & \mathrm{C}(10) & 1.451(2) \\ \mathrm{N}(1) & \mathrm{C}(20) & 1.393(2) & \mathrm{N}(2) & \mathrm{C}(19) & 1.142(2) \\ \mathrm{C}(1) & \mathrm{C}(2) & 1.544(2) & \mathrm{C}(1) & \mathrm{C}(9) & 1.549(2) \\ \mathrm{C}(1) & \mathrm{C}(16) & 1.568(2) & \mathrm{C}(1) & \mathrm{C}(19) & 1.476(2) \\ \mathrm{C}(2) & \mathrm{C}(3) & 1.470(2) & \mathrm{C}(3) & \mathrm{C}(4) & 1.405(2) \\ \mathrm{C}(3) & \mathrm{C}(8) & 1.398(2) & \mathrm{C}(4) & \mathrm{C}(5) & 1.382(2) \\ \mathrm{C}(5) & \mathrm{C}(6) & 1.390(2) & \mathrm{C}(6) & \mathrm{C}(7) & 1.381(2) \\ \mathrm{C}(7) & \mathrm{C}(8) & 1.390(2) & \mathrm{C}(10) & \mathrm{C}(11) & 1.390(2) \\ \mathrm{C}(10) & \mathrm{C}(15) & 1.385(2) & \mathrm{C}(11) & \mathrm{C}(12) & 1.388(2) \\ \mathrm{C}(12) & \mathrm{C}(13) & 1.376(3) & \mathrm{C}(13) & \mathrm{C}(14) & 1.382(3) \\ \mathrm{C}(14) & \mathrm{C}(15) & 1.392(2) & \mathrm{C}(16) & \mathrm{C}(17) & 1.498(2) \\ \mathrm{C}(17) & \mathrm{C}(18) & 1.316(2) & \mathrm{C}(20) & \mathrm{C}(21) & 1.489(2) \\ \mathrm{C}(21) & \mathrm{C}(22) & 1.318(2) & & & \end{array}$

Table 5. Bond lengths involving hydrogens $(\AA)$

$\begin{array}{llllll}\text { atom } & \text { atom } & \text { distance } & \text { atom } & \text { atom } & \text { distance } \\ \mathrm{C}(4) & \mathrm{H}(1) & 0.947 & \mathrm{C}(5) & \mathrm{H}(2) & 0.951 \\ \mathrm{C}(6) & \mathrm{H}(3) & 0.948 & \mathrm{C}(7) & \mathrm{H}(4) & 0.949 \\ \mathrm{C}(9) & \mathrm{H}(5) & 0.945 & \mathrm{C}(11) & \mathrm{H}(6) & 0.951 \\ \mathrm{C}(12) & \mathrm{H}(7) & 0.947 & \mathrm{C}(13) & \mathrm{H}(8) & 0.949 \\ \mathrm{C}(14) & \mathrm{H}(9) & 0.948 & \mathrm{C}(15) & \mathrm{H}(10) & 0.944 \\ \mathrm{C}(16) & \mathrm{H}(11) & 0.947 & \mathrm{C}(16) & \mathrm{H}(12) & 0.947 \\ \mathrm{C}(17) & \mathrm{H}(13) & 0.944 & \mathrm{C}(18) & \mathrm{H}(14) & 0.948 \\ \mathrm{C}(18) & \mathrm{H}(15) & 0.953 & \mathrm{C}(21) & \mathrm{H}(16) & 0.949 \\ \mathrm{C}(22) & \mathrm{H}(17) & 0.944 & \mathrm{C}(22) & \mathrm{H}(18) & 0.952\end{array}$

Table 6. Bond angles (0)

atom atom atom angle atom atom atom angle 


$\begin{array}{llllllll}\mathrm{C}(8) & \mathrm{O}(2) & \mathrm{C}(9) & 118.26(12) & \mathrm{C}(9) & \mathrm{N}(1) & \mathrm{C}(10) & 120.14(12) \\ \mathrm{C}(9) & \mathrm{N}(1) & \mathrm{C}(20) & 116.95(12) & \mathrm{C}(10) & \mathrm{N}(1) & \mathrm{C}(20) & 119.69(12) \\ \mathrm{C}(2) & \mathrm{C}(1) & \mathrm{C}(9) & 112.39(12) & \mathrm{C}(2) & \mathrm{C}(1) & \mathrm{C}(16) & 107.33(12) \\ \mathrm{C}(2) & \mathrm{C}(1) & \mathrm{C}(19) & 110.66(12) & \mathrm{C}(9) & \mathrm{C}(1) & \mathrm{C}(16) & 107.66(12) \\ \mathrm{C}(9) & \mathrm{C}(1) & \mathrm{C}(19) & 109.09(12) & \mathrm{C}(16) & \mathrm{C}(1) & \mathrm{C}(19) & 109.62(12) \\ \mathrm{O}(1) & \mathrm{C}(2) & \mathrm{C}(1) & 121.70(14) & \mathrm{O}(1) & \mathrm{C}(2) & \mathrm{C}(3) & 124.43(14) \\ \mathrm{C}(1) & \mathrm{C}(2) & \mathrm{C}(3) & 113.72(13) & \mathrm{C}(2) & \mathrm{C}(3) & \mathrm{C}(4) & 120.48(14) \\ \mathrm{C}(2) & \mathrm{C}(3) & \mathrm{C}(8) & 120.71(14) & \mathrm{C}(4) & \mathrm{C}(3) & \mathrm{C}(8) & 118.81(14) \\ \mathrm{C}(3) & \mathrm{C}(4) & \mathrm{C}(5) & 120.57(15) & \mathrm{C}(4) & \mathrm{C}(5) & \mathrm{C}(6) & 119.35(16) \\ \mathrm{C}(5) & \mathrm{C}(6) & \mathrm{C}(7) & 121.32(17) & \mathrm{C}(6) & \mathrm{C}(7) & \mathrm{C}(8) & 119.21(16) \\ \mathrm{O}(2) & \mathrm{C}(8) & \mathrm{C}(3) & 123.28(14) & \mathrm{O}(2) & \mathrm{C}(8) & \mathrm{C}(7) & 116.01(14) \\ \mathrm{C}(3) & \mathrm{C}(8) & \mathrm{C}(7) & 120.71(14) & \mathrm{O}(2) & \mathrm{C}(9) & \mathrm{N}(1) & 112.37(11) \\ \mathrm{O}(2) & \mathrm{C}(9) & \mathrm{C}(1) & 110.14(12) & \mathrm{N}(1) & \mathrm{C}(9) & \mathrm{C}(1) & 114.73(12) \\ \mathrm{N}(1) & \mathrm{C}(10) & \mathrm{C}(11) & 120.15(15) & \mathrm{N}(1) & \mathrm{C}(10) & \mathrm{C}(15) & 119.11(14) \\ \mathrm{C}(11) & \mathrm{C}(10) & \mathrm{C}(15) & 120.73(15) & \mathrm{C}(10) & \mathrm{C}(11) & \mathrm{C}(12) & 119.15(18) \\ \mathrm{C}(11) & \mathrm{C}(12) & \mathrm{C}(13) & 120.4(2) & \mathrm{C}(12) & \mathrm{C}(13) & \mathrm{C}(14) & 120.35(19) \\ \mathrm{C}(13) & \mathrm{C}(14) & \mathrm{C}(15) & 120.0(2) & \mathrm{C}(10) & \mathrm{C}(15) & \mathrm{C}(14) & 119.31(17) \\ \mathrm{C}(1) & \mathrm{C}(16) & \mathrm{C}(17) & 114.04(13) & \mathrm{C}(16) & \mathrm{C}(17) & \mathrm{C}(18) & 123.38(17) \\ \mathrm{N}(2) & \mathrm{C}(19) & \mathrm{C}(1) & 178.41(16) & \mathrm{O}(3) & \mathrm{C}(20) & \mathrm{N}(1) & 121.37(15) \\ \mathrm{O}(3) & \mathrm{C}(20) & \mathrm{C}(21) & 122.18(15) & \mathrm{N}(1) & \mathrm{C}(20) & \mathrm{C}(21) & 116.40(14) \\ \mathrm{C}(20) & \mathrm{C}(21) & \mathrm{C}(22) & 120.85(17) & & & & \end{array}$

Table 7. Bond angles involving hydrogens ( $(0)$

$\begin{array}{llllllll}\text { atom } & \text { atom } & \text { atom } & \text { angle } & \text { atom } & \text { atom } & \text { atom } & \text { angle } \\ \mathrm{C}(3) & \mathrm{C}(4) & \mathrm{H}(1) & 119.5 & \mathrm{C}(5) & \mathrm{C}(4) & \mathrm{H}(1) & 120.0 \\ \mathrm{C}(4) & \mathrm{C}(5) & \mathrm{H}(2) & 120.9 & \mathrm{C}(6) & \mathrm{C}(5) & \mathrm{H}(2) & 119.8 \\ \mathrm{C}(5) & \mathrm{C}(6) & \mathrm{H}(3) & 119.0 & \mathrm{C}(7) & \mathrm{C}(6) & \mathrm{H}(3) & 119.6 \\ \mathrm{C}(6) & \mathrm{C}(7) & \mathrm{H}(4) & 120.4 & \mathrm{C}(8) & \mathrm{C}(7) & \mathrm{H}(4) & 120.4 \\ \mathrm{O}(2) & \mathrm{C}(9) & \mathrm{H}(5) & 106.5 & \mathrm{~N}(1) & \mathrm{C}(9) & \mathrm{H}(5) & 106.5 \\ \mathrm{C}(1) & \mathrm{C}(9) & \mathrm{H}(5) & 106.0 & \mathrm{C}(10) & \mathrm{C}(11) & \mathrm{H}(6) & 120.0 \\ \mathrm{C}(12) & \mathrm{C}(11) & \mathrm{H}(6) & 120.8 & \mathrm{C}(11) & \mathrm{C}(12) & \mathrm{H}(7) & 120.0 \\ \mathrm{C}(13) & \mathrm{C}(12) & \mathrm{H}(7) & 119.6 & \mathrm{C}(12) & \mathrm{C}(13) & \mathrm{H}(8) & 120.2 \\ \mathrm{C}(14) & \mathrm{C}(13) & \mathrm{H}(8) & 119.4 & \mathrm{C}(13) & \mathrm{C}(14) & \mathrm{H}(9) & 119.9 \\ \mathrm{C}(15) & \mathrm{C}(14) & \mathrm{H}(9) & 120.1 & \mathrm{C}(10) & \mathrm{C}(15) & \mathrm{H}(10) & 119.7 \\ \mathrm{C}(14) & \mathrm{C}(15) & \mathrm{H}(10) & 121.0 & \mathrm{C}(1) & \mathrm{C}(16) & \mathrm{H}(11) & 107.9\end{array}$




$\begin{array}{llllllll}\mathrm{C}(1) & \mathrm{C}(16) & \mathrm{H}(12) & 107.9 & \mathrm{C}(17) & \mathrm{C}(16) & \mathrm{H}(11) & 108.4 \\ \mathrm{C}(17) & \mathrm{C}(16) & \mathrm{H}(12) & 108.6 & \mathrm{H}(11) & \mathrm{C}(16) & \mathrm{H}(12) & 109.9 \\ \mathrm{C}(16) & \mathrm{C}(17) & \mathrm{H}(13) & 118.0 & \mathrm{C}(18) & \mathrm{C}(17) & \mathrm{H}(13) & 118.7 \\ \mathrm{C}(17) & \mathrm{C}(18) & \mathrm{H}(14) & 119.8 & \mathrm{C}(17) & \mathrm{C}(18) & \mathrm{H}(15) & 120.2 \\ \mathrm{H}(14) & \mathrm{C}(18) & \mathrm{H}(15) & 120.0 & \mathrm{C}(20) & \mathrm{C}(21) & \mathrm{H}(16) & 119.5 \\ \mathrm{C}(22) & \mathrm{C}(21) & \mathrm{H}(16) & 119.6 & \mathrm{C}(21) & \mathrm{C}(22) & \mathrm{H}(17) & 120.5 \\ \mathrm{C}(21) & \mathrm{C}(22) & \mathrm{H}(18) & 119.1 & \mathrm{H}(17) & \mathrm{C}(22) & \mathrm{H}(18) & 120.4\end{array}$

